\title{
Airway angiopoietin-2 in ventilated very preterm infants: association with prenatal factors and neonatal outcome
}

\begin{tabular}{|c|c|}
\hline Journal: & Pediatric Pulmonology \\
\hline Manuscript ID: & PPUL-10-0020.R2 \\
\hline Wiley - Manuscript type: & Original Article \\
\hline $\begin{array}{l}\text { Date Submitted by the } \\
\text { Author: }\end{array}$ & $11-$ Nov-2010 \\
\hline Complete List of Authors: & $\begin{array}{l}\text { Thomas, Wolfgang; University of Wuerzburg, Department of } \\
\text { Pediatrics } \\
\text { Seidenspinner, Silvia; University of Wuerzburg, Department of } \\
\text { Pediatrics } \\
\text { Kramer, B; Akademisch Ziekenhuis Maastricht, Department of } \\
\text { Pediatrics } \\
\text { wirbelauer, johannes; University of Wuerzburg, Department of } \\
\text { Pediatrics } \\
\text { Kawczyńska-Leda, Natalia; Poznań University of Medical Sciences, } \\
\text { Department of Neonatology } \\
\text { Szymankiewicz, Marta; Poznań University of Medical Sciences, } \\
\text { Department of Neonatology } \\
\text { Speer, Christian; University of Wuerzburg, Department of Pediatrics }\end{array}$ \\
\hline Keywords: & $\begin{array}{l}\text { bronchopulmonary dysplasia, angiogenesis, small for gestational } \\
\text { age infant, vascular endothelial growth factor, lung injury }\end{array}$ \\
\hline
\end{tabular}

\section{SCHOLARONE Manuscripts}


1 Airway angiopoietin-2 in ventilated very preterm infants: association with prenatal factors and

2 neonatal outcome

3 Wolfgang Thomas ${ }^{1}$, Silvia Seidenspinner ${ }^{1}$, Boris W. Kramer ${ }^{2}$, Johannes Wirbelauer ${ }^{1}$, Natalia

4 Kawczyńska-Leda ${ }^{3}$, Marta Szymankiewicz ${ }^{3}$, Christian P. Speer ${ }^{1}$

5

$6 \quad{ }^{1}$ University Children's Hospital, University of Würzburg, Germany

$7 \quad{ }^{2}$ Department of Pediatrics, Maastricht University Medical Center, Maastricht, The

8 Netherlands

$9 \quad{ }^{3}$ Department of Neonatology, Poznań University of Medical Sciences, Poznań, Poland 10

12 This work was supported in part by a personal grant from G. Bierich, managing director,

13 Interfinanz, Duesseldorf, Germany

14 No author has competing interests to declare. 15

16 Key words: bronchopulmonary dysplasia, lung injury, angiogenesis,

17 vascular endothelial growth factor, small for gestational age infant 18

19 Corresponding author: Wolfgang Thomas, MD; University Children's Hospital, Josef-

20 Schneider-Str. 2, 97080 Würzburg, Germany, Tel.: +49(0)931-20127728,

21 Fax.: +49(0)931-20127714, e-mail: Thomas_w@klinik.uni-wuerzburg.de

22

23 Abbreviated title: Angiopoietin-2 in airways of ventilated preterm infants 
W. Thomas: Angiopoietin-2 in airways of ventilated preterm infants $\quad 2 / 22$

\section{Abstract}

2 Objective: Pulmonary angiogenesis is a prerequisite for lung development. Angiopoietin-2

3 (Ang2) destabilizes endothelial cells through its endothelial receptor TIE-2, enabling vascular

4 sprouting. Ang1 stabilizes new blood vessels. Soluble TIE-2 (sTIE-2) modulates these effects.

5 We hypothesized that histological funisitis is associated with alterations of Ang2 in airways

6 and of the systemic angiopoietin-TIE-2 homeostasis in very low birth weight infants

7 (VLBW), contributing to pulmonary morbidity and mortality.

8 Methods: We measured Ang2 in tracheobronchial aspirate fluid (TAF) of 42 VLBW $<30$

9 weeks of gestation from day 1 through 15 and Ang1, Ang2 and sTIE-2 in umbilical cord

10 serum of 28 infants by ELISA. Histological examination distinguished 3 groups: funisitis,

11 chorioamnionitis and controls.

12 Results: Funisitis was associated with lower Ang2 values in TAF but not with changes of 13 Ang1, Ang2 and sTIE-2 in umbilical cord serum. Infants who developed BPD or died had a 14 persistently decreased ratio of previously measured Ang1 to Ang2 in TAF on days 1-5 and 15 increased cord serum concentrations of sTIE-2. Moderate BPD/death was associated with an 16 increase of Ang2 in TAF on day 10 and decreased Ang1/Ang2 ratio from day 3-15. Small for 17 gestational age (SGA) infants had increased Ang2 in TAF on day 1-7 and a lower Ang1/Ang2 18 ratio on days 5-7.

19 Conclusions: The predominance of Ang2 in airway fluid of infants with BPD/death and SGA 20 infants suggests a link between disrupted placental and fetal pulmonary angiogenesis.

21 Histological funisitis with reduced Ang2 in TAF was of minor relevance for outcome in our 22 cohort. 
W. Thomas: Angiopoietin-2 in airways of ventilated preterm infants $\quad 3 / 22$

\section{Introduction}

2 Angiogenesis plays a pivotal role in organ development and maturation ${ }^{1}$. In extremely

3 premature infants disturbed pulmonary angiogenesis accompanies alveolar simplification,

4 eventually leading to bronchopulmonary dysplasia (BPD) $)^{2-3}$.

5 Angiopoietin-2 (Ang2) belongs to a family of angiogenic growth factors which bind

6 specifically to the tyrosine kinase receptor with immunoglobulin and epidermal growth factor

7 homology domain 2 (TIE-2) on endothelial cells. Ang2 destabilizes the endothelium by

8 decreasing the phosphorylation status of TIE-2, leading to a more responsive endothelium, a

9 prerequisite for sprouting of new vessels ${ }^{4}$. The endothelial destabilization also leads to

10 vascular leakage which is a feature of inflammation ${ }^{5}$. Binding of the Ang2 antagonist

11 angiopoietin-1 (Ang1) to TIE-2 induces tightening of endothelial intercellular junctions,

12 leading to stabilization and maturation of newly formed vessels ${ }^{6}$. Both angiopoietins act

13 synergistically in the process of angiogenesis ${ }^{4}$. A soluble form of TIE-2 (sTIE-2), released

14 from the endothelium after proteolytical cleavage of the receptor's extracellular domain,

15 interferes with the angiopoietin-TIE-2 signaling by binding the free ligands ${ }^{7}$.

16 A differential spatial and temporal regulation of TIE-2 by Ang1 and Ang2 in the lung could

17 be shown a prerequisite for a stable pulmonary vasculature and normal lung development and 18 maturation in a murine model ${ }^{8}$. Increased concentrations of Ang2 in tracheobronchial aspirate

19 fluid (TAF) have been associated with the development of BPD or death in ventilated

20 premature infants 9 .

21 The role of angiopoietins and TIE-2 in systemic inflammatory processes and pulmonary

22 injury has been extensively addressed. In a pediatric population, high plasma Ang2 levels and 23 low Ang1 levels were associated with septic shock ${ }^{10}$. Serum concentrations of sTIE-2 have

24 been shown elevated in adult septic patients at risk for adult respiratory distress syndrome

25 compared to non-septic patients ${ }^{11}$. However, data about blood levels of the angiopoietins and

26 their receptor in very premature infants exposed to prenatal inflammation do not exist. 
W. Thomas: Angiopoietin-2 in airways of ventilated preterm infants $\quad$ 4/22

1 Histological funisitis is regarded as morphological counterpart of a fetal systemic

2 inflammatory response $\mathrm{e}^{12}$ and has been shown associated with down-regulation of angiogenic

3 factors in placental tissue ${ }^{13}$. This fetal inflammatory response has been identified as risk factor

4 for $\mathrm{BPD}^{14}$ and mortality ${ }^{15}$ after premature birth.

5 The primary aim of our exploratory pilot study was to assess the association between

6 histological funisitis and postnatal airway concentrations of Ang2 in ventilated very

7 premature infants. We also investigated concentrations of the factors Ang1, Ang2 and sTIE-2

8 in umbilical cord blood. We speculated that alterations of pulmonary Ang2 and a disturbed

9 homeostasis of the angiopoietin-TIE-2 system after funisitis might contribute to pulmonary

10 morbidity and neonatal mortality of this high risk group of patients. 
W. Thomas: Angiopoietin-2 in airways of ventilated preterm infants $\quad 5 / 22$

\section{$1 \quad$ Material and Methods}

\section{Patients}

342 infants with a birth weight $<1500 \mathrm{~g}$ and a gestational age $<30$ weeks, who were admitted

4 to the intensive care unit of the Department of Neonatology, Poznan University of Medical

5 Sciences, Poland, between November 2004 and August 2005, were enrolled in this

6 prospective study after informed consent of the parents. Patients were inborns, had to be

7 mechanically ventilated for respiratory failure within the first 48 hours of life and had no

8 major congenital malformations. Respiratory support was adjusted to reach a $\mathrm{SpO}_{2}$ between

$985 \%$ and $94 \%$. Infants with supplemental oxygen for at least 28 days were assigned to have

$10 \mathrm{BPD}^{16}$. Depending on the need for oxygen or respiratory support at a postmenstrual age of 36

11 weeks, BPD was graded as mild, moderate or severe ${ }^{16}$. The study was approved by the Ethical

12 Committees of the Poznan University of Medical Sciences and the Faculty of Medicine,

13 University of Wuerzburg, Germany.

\section{Histological examinations}

16 For each infant at least 2 sections of umbilical cord, 2 sections of placental parenchyma and 1

17 section of membrane rolls were evaluated for the presence and severity of neutrophilic

18 infiltrates by two pathologists, unaware of clinical data, according to a protocol ${ }^{17}$. Patients

19 were assigned to three groups according to histological diagnosis. The first group was

20 characterized by chorioamnionitis and funisitis and will be referred to as funisitis group

21 ( $\mathrm{n}=17)$. In the second group chorioamnionitis without funisitis was diagnosed

22 (chorioamnionitis group, $\mathrm{n}=9$ ). The control group consisted of 16 patients without histological 23 signs of inflammation in placenta, membranes and umbilical cord. 
W. Thomas: Angiopoietin-2 in airways of ventilated preterm infants $\quad 6 / 22$

\section{Sampling, preparation and storage}

2 Tracheobronchial aspiration was performed in a standardized manner up to 3 times per day on

3 days $1,3,5,7,10$ and 15 of life. After instillation of $0.5 \mathrm{ml} \mathrm{NaCl} 0.9 \%$ into the endotracheal

4 tube a suction catheter was inserted slightly beyond the distal tip of the tube and airway

5 secretions were suctioned and collected in sterile mucus traps (Unomedical, Birkeroed,

6 Denmark). Each specimen was diluted with $\mathrm{NaCl} 0.9 \%$ to a volume of $0.5 \mathrm{ml}$. Specimens of

7 one day were pooled. They were spun at 3,000 revolutions per minute (rpm) for 5 minutes.

8 Supernatants were centrifuged again twice at 10,000 rpm for 5 minutes. Specimens were

9 cooled during processing, including centrifugation. The resulting clear supernatants

10 were divided into aliquots and stored at $-80^{\circ} \mathrm{C}$ until further analysis.

11 Umbilical cord blood could be collected from the umbilical cord vein of 28 infants. Blood 12 was centrifuged at $3,000 \mathrm{rpm}$ for 10 minutes and the serum stored at $-80^{\circ} \mathrm{C}$ until analysis.

14 Measurement of factors and secretory component for IgA

15 Concentrations of Ang1, Ang2 and sTIE-2 in umbilical cord serum and levels of Ang2 in 16 TAF were measured with commercially available enzyme-linked immunosorbent assays 17 (ELISA) (R\&D Systems, Minneapolis, USA). To correct for different dilutions, 18 concentrations in TAF were related to the secretory component for IgA (SC). SC levels in 19 TAF of preterm infants are largely independent of capillary leakage, gestational and postnatal 20 age as well as respiratory disease in the first weeks of life which makes it a valid dilutional 21 marker of epithelial lining fluid ${ }^{18-19}$. SC was measured by ELISA as previously described ${ }^{21}$.

23 Statistical analysis

24 Continuous variables were analyzed with one-way analysis of variance (ANOVA), if they 25 showed a normal distribution within groups and homogeneity of variance. Data with a skewed 
W. Thomas: Angiopoietin-2 in airways of ventilated preterm infants

1 distribution were primarily assessed with nonparametric tests (Kruskal-Wallis test, Mann-

2 Whitney-U-test). We used post hoc Bonferroni method to correct for multiple comparisons.

3 Sequential values were compared with Friedman test and categorical or nominal values

4 assessed with $\chi^{2}$-test. Correlations were analyzed using Spearman's rank correlation

5 coefficients. All tests were two-sided, a value of $\mathrm{p}<0.05$ was considered significant. Statistical

6 evaluation was performed with SPSS 18.0 for Windows (SPSS Inc., Chicago, USA). 
W. Thomas: Angiopoietin-2 in airways of ventilated preterm infants $\quad 8 / 22$

\section{Results}

\section{Patients}

3 Clinical characteristics and outcome of patients in the 3 groups control, chorioamnionitis and

4 funisitis are summarized in table 1. Infants of the chorioamnionitis group had lower birth

5 weights than those in the control and funisitis group $(\mathrm{p}=0.01)$. Gestational age and proportions

6 of small for gestational age infants (SGA, i.e. birth weight $<10$. percentile for gestational age

7 and gender) did not differ significantly between groups. Most infants born after maternal

8 clinical signs of amnion infection and premature rupture of membranes belonged to the

9 funisitis group. The initial blood leukocyte count was higher and the interval between rupture

10 of membranes and birth was longer in the funisitis group compared to chorioamnionitis and

11 control $(\mathrm{p}<0.01$, respectively).

12 Thirty-six infants had received prenatal steroids. Within the first 15 days of life a single dose

13 of hydrocortisone was administered to 4 infants (days 1, 3, 6 and 9 respectively) and a single

14 dose of dexamethasone was given to 2 infants (day 8 and 10, respectively). Twenty-six infants

15 were treated with a natural surfactant preparation up to 3 times. Administration of prenatal

16 and postnatal corticosteroids or surfactant did not differ between groups.

17 There was no difference in respiratory outcome measures, such as duration of mechanical

18 ventilation, incidence of BPD or duration of oxygen therapy, between groups. TAF was

19 cultured for Ureaplasma urealyticum in 40 infants. Positive cultures (11) did not differ

20 between groups and were not associated with respiratory outcome either.

21 Mortality was highest in the chorioamnionitis group. Overall, 10 infants of the study cohort

22 died until day of life 25 . They had a significantly lower gestational age (mean \pm standard

23 deviation: $25.7 \pm 1.7$ weeks vs. $27.9 \pm 1.5$ weeks, $\mathrm{p}<0.01)$ and birth weight $(857 \pm 256 \mathrm{~g}$ vs.

$241067 \pm 199 \mathrm{~g}, \mathrm{p}<0.05)$ than survivors and were all mechanically ventilated from the day of

25 birth until death. 
W. Thomas: Angiopoietin-2 in airways of ventilated preterm infants $\quad 9 / 22$

1 Nineteen infants developed BPD, 13 had mild, 6 moderate and no infant severe BPD. Infants,

2 who developed BPD or died, had a lower gestational age and birth weight and were more

3 likely to be males ( $\mathrm{p}<0.05$, respectively) than those who survived without BPD. They were

4 mechanically ventilated for a longer period $(\mathrm{p}<0.01)$ and needed higher peak oxygen

5 supplementation as well as higher peak inspiratory pressure on mechanical ventilation

6 ( $\mathrm{p}<0.05$, respectively, table 2). All 6 SGA infants either developed BPD (5) or died (1).

7

8 Ang2/SC in $T A F$

9 Ang2/SC in TAF of the 42 infants decreased over time. Day 1, median: $110.4 \mathrm{pg} / \mu \mathrm{g}$

10 (interquartile range: 44.1-218.9); day 3, $71.8 \mathrm{pg} / \mu \mathrm{g}$ (44.3-106.6); day 5, $39.8 \mathrm{pg} / \mu \mathrm{g}(21.3-$

11 114.0); day 7, $30.1 \mathrm{pg} / \mu \mathrm{g}(16.9-62.4)$; day $10,32.1 \mathrm{pg} / \mu \mathrm{g}(16.9-49.0)$ and day $15,23.8 \mathrm{pg} / \mu \mathrm{g}$

$12 \quad(7.7-35.6) ; \mathrm{p}<0.001$.

13 Figure 1 illustrates values for Ang2/SC in the 3 groups control, chorioamnionitis and funisitis.

14 The funisitis group had significantly lower values in TAF than the control group on days 1-3

$15(\mathrm{p}<0.01)$ and $10(\mathrm{p}<0.05)$ and lower values than the chorioamnionitis group on days 1-7 $(\mathrm{p}<$

160.01 , respectively), whereas Ang2/SC did not differ significantly between the

17 chorioamnionitis and control group on any day.

18 Infants who developed a moderate form of BPD or died showed significantly higher Ang2/SC

19 on day $10(49.5 \mathrm{pg} / \mu \mathrm{g}$ [38.2-72.5]) compared to the remainder (24.2 pg/ $\mu \mathrm{g}$ [15.8-36.5], p

$20<0.05)$.

21 Ang2/SC did not correlate with gestational age and did not differ between genders. On days

22 1-7 SGA infants had significantly higher Ang2/SC levels than those infants whose birth

23 weight was appropriate for gestational age (AGA) (figure 2). Surfactant therapy, pre- and

24 postnatal corticosteroids and colonization with Ureaplasma urealyticum were not associated

25 with alterations of Ang2/SC. 
W. Thomas: Angiopoietin-2 in airways of ventilated preterm infants $\quad 10 / 22$

\section{$1 \quad$ Ratio Ang $1 /$ Ang2}

2 We used TAF concentrations of the Ang2 counterpart Ang1, which we had previously

3 measured in the same cohort, to calculate the ratio between the two factors ${ }^{32}$. The Ang1/Ang2

4 ratio did not differ significantly between the three groups control, chorioamnionitis and

5 funisitis (data not shown).

6 On days 1-5, the ratio was significantly lower in infants who developed BPD or died $(\mathrm{p}<0.05$,

7 respectively). Patients, who developed moderate BPD or died, had significantly lower values

8 on days 3-15 compared to the remaining infants ( $\mathrm{p}<0.05$, respectively) (figure 3 ). The ratio

9 was significantly decreased in SGA infants compared to AGA infants on days 5 and 7 (p<

$100.05)$.

11 Umbilical cord serum concentrations

12 Umbilical cord blood could be obtained from 28 infants of our cohort. They did not differ

13 significantly in any demographic or outcome parameter from the remaining 14 infants.

14 Umbilical cord serum concentrations of Ang1, Ang2 and sTie-2 were not different between

15 the 3 groups control, chorioamnionitis and funisitis. They did not differ between SGA and

16 AGA infants either. Patients, who developed BPD or died, had significantly higher sTIE-2

17 concentrations $(\mathrm{p}<0.05)$ than those that survived without BPD (figure 4). 
W. Thomas: Angiopoietin-2 in airways of ventilated preterm infants $\quad$ 11/22

\section{Discussion}

2 In our exploratory study histological funisitis was associated with decreased concentrations of

3 Ang2 in TAF of ventilated very premature infants compared to infants exposed to

4 chorioamnionitis or to no inflammation in utero. Pulmonary angiogenesis is a prerequisite for

5 alveolarisation ${ }^{2,21}$ and Ang2 is responsible for endothelial cell sprouting and promotes

6 vascular remodeling ${ }^{22}$. In vitro, proangiogenic signaling of Ang2 by activation of TIE-2 has

7 been shown concentration dependent ${ }^{23}$. One might therefore speculate that decreased

8 proangiogenic signaling by Ang2 in the lungs of very premature infants with funisitis -

9 reflecting a fetal systemic inflammatory response ${ }^{12}$ - may predispose them for disturbed

10 pulmonary angiogenesis along with impaired alveolar development, eventually contributing to

11 the risk of $\mathrm{BPD}^{14-15}$.

12 However, we found higher Ang2 concentrations in TAF of ventilated VLBW infants on day

1310 to be associated with the outcome moderate BPD or death along with a reduced ratio of

14 Ang1 to Ang2 in TAF on many of the first postnatal days in infants who developed mild to

15 moderate BPD or died. These findings suggest that a preponderance of Ang2 in airway fluid

16 of ventilated very premature infants plays a role for pulmonary outcome and neonatal

17 mortality. Increased Ang2 concentrations in TAF in the first week of life of ventilated VLBW

18 infants have recently been shown associated with the combined outcome moderate BPD or

19 death ${ }^{9}$. Our findings confirm and amend these data.

20 In recent years, the association between histological funisitis and respiratory morbidity in very

21 premature infants has been questioned ${ }^{24-26}$. In our cohort funisitis was not associated with the

22 outcome BPD or death. This may be due to a lack of power to detect such an association with

23 our samples size. Moreover, funisitis does not represent a homogeneous disease ${ }^{27}$. Its risk is

24 modified by factors, like duration of membrane rupture, which by themselves interfere with

25 neonatal outcome. Need for sustained mechanical ventilation and higher oxygen 
W. Thomas: Angiopoietin-2 in airways of ventilated preterm infants

$12 / 22$

1 between prenatal inflammation and BPD ${ }^{26,28-29}$, were both associated with the combined

2 outcome BPD or death in our cohort. Hyperoxia has been shown to induce pulmonary Ang2

3 expression on transcriptional and translational level in an animal model of acute lung injury

4 and to decrease Ang1 mRNA expression simultaneously ${ }^{30}$. In this setting the abundance of

5 Ang2 seems to play an important role for pulmonary oxidative injury, inflammation and cell

6 death $^{30}$. These experimental data are also in accordance with our findings and underscore the

7 relevance of postnatal factors as potential risk modulators for $\mathrm{BPD}^{28}$.

8 Treatment strategies targeting to reduce pulmonary Ang2 expression may have potential

9 benefits for respiratory outcome ${ }^{30}$. Ang2 concentrations in TAF of VLBW infants have been

10 decreased after administration of dexamethasone ${ }^{9}$. However, our data suggest that the overall

11 balance between different types of factors - i.e. between Ang1, which stabilizes endothelium

12 and leads to vascular maturation ${ }^{6}$, and Ang2, which destabilizes vascular endothelium ${ }^{4-5}$ - may

13 be as important for respiratory outcome of very premature infants as changes in one single

14 factor. This is in accordance with data from a model of lipopolysaccharide-induced acute lung

15 injury $^{31}$. Likewise, we have recently demonstrated an imbalance between Ang1 and the

16 angiostatic factor endostatin in TAF of extremely premature infants to be associated with the

17 combined outcome BPD or death ${ }^{32}$.

18 The functions of angiopoietins are pleiotropic and context-dependent ${ }^{1}$. Ang2 synergizes with

19 vascular endothelial growth factor (VEGF) to stimulate angiogenesis but causes endothelial

20 cell death and vessel regression in the absence of sufficient angiogenic signals ${ }^{1}$. VEGF has

21 been shown decreased in TAF of ventilated very premature infants developing BPD and in

22 pulmonary tissue of infants who had died from the disease ${ }^{33-35}$. In light of these data our

23 findings would support the concept of impaired vascular signaling in the pathogenesis of

24 BPD.

25 Interestingly, Ang2 levels in TAF of SGA infants were increase compared to AGA infant

26 during the first week of life in our cohort, along with an imbalance between Ang1 and Ang2 
1 on day 5 and 7. All SGA infants either developed BPD or died. These findings are in line with

22 recent studies, which identified preeclampsia and intrauterine growth retardation (IUGR),

3 but not histological chorioamnionitis with funisitis, as risk factors for BPD in extremely

4 premature infants ${ }^{24,36}$.

5 Regulation of vascular development in the feto-maternal unit is disturbed in preeclampsia,

6 leading to disturbed placentation and eventually to IUGR. Similarly, disrupted pulmonary

7 vascularisation plays a crucial role for $\mathrm{BPD}^{2,3,21}$. Our data complement existing evidence that

8 an imbalance between Ang1 and Ang2 in different feto-maternal compartments is a common

9 denominator of impaired placental as well as fetal pulmonary vascular development. In

10 maternal plasma at midgestation, IUGR has been found associated with increased Ang2

11 concentrations $^{37}$ and preeclampsia with a decrease of the Ang1/Ang2 ratio $^{38}$. A decrease of

12 Ang2 concentrations along with an increase of the Ang1/Ang2 ratio in the amniotic cavity

13 seems to be critical to prevent regression, destabilization and leakage of placental vessels

14 during normal gestation ${ }^{39-40}$. Intraamniotic Ang2 might be at least partially of fetal pulmonary

15 origin $^{30,39}$. Higher Ang2 level in TAF during the initial phase of life in SGA infants might

16 therefore reflect both disturbed placental and fetal pulmonary angiogenesis ${ }^{41}$.

17 Remarkably, intraamniotic inflammation/infection - assessed by either the presence of soluble

18 markers of the innate immune system, increased intraamniotic IL-6 or positive amniotic fluid

19 cultures - have been shown associated with higher intraamniotic Ang2 levels in women

20 presenting with preterm labor or preterm premature rupture of membranes in 2 studies ${ }^{39-40}$.

21 Both studies failed to demonstrate an association between histological funisitis and higher

22 intraamniotic Ang2. In placentas of very premature infants angiogenic factors have been

23 demonstrated to be decreased after histological chorioamnionitis and funisitis ${ }^{13}$. However,

24 histological evidence of inflammation was not associated with morphological alterations of

25 the placental vasculature in this study. These data underscore a limited relevance of

26 histological chorioamionitis with funisitis for angiogenic homeostasis in maternal and fetal 
W. Thomas: Angiopoietin-2 in airways of ventilated preterm infants

1 tissues. Likewise, histological funisitis was not associated with changes of umbilical cord

2 serum concentrations of Ang1, Ang2 and sTIE-2 concentrations in our cohort.

3 However, sTIE-2 levels in umbilical cord serum were higher in infants who developed BPD

4 or died. In critically ill adult patients concentrations of sTIE-2 in blood have been associated

5 with increased pulmonary vascular permeability ${ }^{11}$, which plays a role in the early phase of

$6 \mathrm{BPD}^{16,20,29}$. sTIE-2 is released into the blood after proteolytical cleavage of the extracellular

7 domain of the endothelial receptor, either constitutively or after proangiogenic stimulation ${ }^{7,42}$.

8 sTIE-2 is believed to act as a decoy for angiopoietins, affecting the signaling pathways

9 induced by binding of the ligands to the membrane-bound receptor ${ }^{42}$. As Ang1 has been

10 shown to bind to sTIE-2 with a much higher affinity than Ang2 in vitro ${ }^{43}$, high sTIE-2

11 concentrations may shift the Ang1/Ang2 ratio in favour of Ang2, eventually contributing to

12 systemic and pulmonary endothelial activation and destabilization ${ }^{11}$.

13 Lower gestational and birth weight as well as sustained mechanical ventilation of infants who

14 died in our cohort represent important risk factors for all grades of BPD. We therefore

15 combined the outcome BPD and death for analysis. The higher mortality in the

16 chorioamnionitis group compared to both other groups can be attributed to the lower birth

17 weight in this group as important predictor of mortality in VLBW infants ${ }^{44}$.

18 Our clinical explorative study has limitations. We did not assess the activity but only

19 concentrations of factors involved in angiogenesis. Moreover, our sample size made inclusion

20 of potential confounders in a multivariate analysis not applicable as the power to detect

21 associations between independent factors and concentrations of the angiogenic factors would

22 have been less than $80 \%$ in most cases. Therefore, our study can only be regarded preliminary

23 and suitable to generate new hypotheses, which have to be proved by experimental studies

24 and clinical studies with appropriate patient numbers to allow multivariate comparisons,

25 before definite conclusions can be drawn. 
W. Thomas: Angiopoietin-2 in airways of ventilated preterm infants $\quad 15 / 22$

1 To conclude, our data suggest that an increase of Ang2 and an imbalance with its counterpart

2 Ang1 in airway fluid potentially contributes to a sustained disturbance of pulmonary vascular

3 and alveolar development in ventilated very preterm infants who develop BPD or die. The

4 presence of such an imbalance in SGA infants implies an association between disrupted

5 placental and fetal pulmonary angiogenesis. We demonstrated that histological funisitis

6 associated with reduced Ang2 concentrations in airway fluid is of minor relevance for

7 outcome. Further ante- and postnatal factors are likely to modulate the risk of disturbed

8 pulmonary vascularisation and alveolarisation in very premature infants.

9

10 Acknowledgement

11 We thank Alexander Marx, Institute of Pathology, University Hospital Mannheim, Germany

12 and Maria Chmielnicka-Kopaczyk, Department of Pathology, Poznań University of Medical

13 Sciences, Poznań, Poland for histological evaluation and Daniela Keller, Department of

14 Bioinformatics, University of Wuerzburg, Germany, for statistical advice.

17 No author has competing interests to declare. 
W. Thomas: Angiopoietin-2 in airways of ventilated preterm infants $\quad 16 / 22$

1 References

2

3 1. Carmeliet P. Angiogenesis in health and disease. Nature Med 2003;9:653-660.

42 2. Thebaud B. Angiogenesis in lung development, injury and repair: implications for

$5 \quad$ chronic lung disease of prematurity. Neonatology 2007;9:291-297.

6 3. Stenmark KR, Abman SH. Lung vascular development: implications for the pathogenesis of bronchopulmonary dysplasia. Ann Rev Physiol 2005;67:623-661.

8 4. Distler JH, Hirth A, Kurowska-Stolarska M, Gay RE, Gay S, Distler O. Angiogenic and angiostatic factors in the molecular control of angiogenesis. Q J Nucl Med 2003;47:149-161.

11 5. Roviezzo F, Tsigkos S, Kotanidou A, Bucci M, Brancaleone V, Cirino G,

Papapetropoulos A. Angiopoietin-2 causes inflammation in vivo by promoting vascular leakage. J Pharmacol Exp Ther 2005;314:738-744.

6. Makinde T, Agrawal DK. Intra and extravascular transmembrane signalling of

7. Findley CM, Cudmore MJ, Ahmed A, Kontos CD. VEGF Induces Tie2 shedding via a phosphoinositide 3-kinase/Akt dependent pathway to modulate Tie2 signaling. Arterioscler Thromb Vasc Biol 2007;27:2619-2626.

19 8. Hato T, Kimura Y, Morisada T, Koh GY, Miyata K, Tabata M, Kadomatsu T, Endo

23 9. Aghai ZH, Faqiri S, Saslow JG, Nakhla T, Farhath S, Kumar A, Eydelman R, Strande L, Stahl G, Leone P, Bhandari V. Angiopoietin 2 concentrations in infants developing bronchopulmonary dysplasia: attenuation by dexamethasone. J Perinatol 2008;28:149155. 
W. Thomas: Angiopoietin-2 in airways of ventilated preterm infants $\quad$ 17/22

1 10. Giuliano JS, Jr., Lahni PM, Harmon K, Wong HR, Doughty LA, Carcillo JA,

2

3

4

Zingarelli B, Sukhatme VP, Parikh SM, Wheeler DS. Admission angiopoietin levels in children with septic shock. Shock 2007;28:650-654.

11. van der Heijden M, van Nieuw Amerongen GP, van Hinsbergh VW, Groeneveld AJ. The interaction of soluble Tie2 with angiopoietins and pulmonary vascular permeability in septic and non-septic critically ill patients. Shock 2010;33:263-268.

12. Pacora P, Chaiworapongsa T, Maymon E, Kim YM, Gomez R, Yoon BH, Ghezzi F, Berry SM, Qureshi F, Jacques SM, Kim JC, Kadar N, Romero R. Funisitis and chorionic vasculitis: the histological counterpart of the fetal inflammatory response syndrome. J Matern Fetal Neonatal Med 2002;11:18-25.

13. Kramer BW, Kaemmerer U, Kapp M, Herbst D, Marx A, Berg D, Groneck PA, Speer CP. Decreased expression of angiogenic factors in placentas with chorioamnionitis after preterm birth. Pediatr Res 2005;58:607-612.

14. Yoon BH, Romero R, Kim KS, Park JS, Ki SH, Kim BI, Jun JK. A systemic fetal inflammatory response and the development of bronchopulmonary dysplasia. Am J Obstet Gynecol 1999;181:773-779.

15. Lau J, Magee F, Qiu Z, Hoube J, Von Dadelszen P, Lee SK. Chorioamnionitis with a fetal inflammatory response is associated with higher neonatal mortality, morbidity, and resource use than chorioamnionitis displaying a maternal inflammatory response only. Am J Obstet Gynecol 2005;193:708-713.

16. Jobe AH, Bancalari E. Bronchopulmonary dysplasia. Am J Respir Crit Care Med 2001;163(7):1723-1729.

17. Rogers BB, Alexander JM, Head J, McIntire D, Leveno KJ. Umbilical vein interleukin-6 levels correlate with the severity of placental inflammation and gestational age. Hum Pathol 2002;33:335-340. 
W. Thomas: Angiopoietin-2 in airways of ventilated preterm infants $\quad 18 / 22$

1 18. Dargaville PA, South M, Vervaart P, McDougall PN. Validity of markers of dilution

2

3

4

5

6

7

8

9

10

11

12

13

14

15

16

17

18

19

20

21

22

23

24 in small volume lung lavage. Am J Respir Crit Care Med 1999;16:778-784.

19. Watts CL, Bruce MC. Comparison of secretory component for immunoglobulin A with albumin as reference proteins in tracheal aspirate from preterm infants. J Pediatr 1995; 127:113-122.

20. Groneck P, Götze-Speer B, Oppermann M, Eiffert H, Speer CP. Association of pulmonary inflammation and increased microvascular permeability during the development of bronchopulmonary dysplasia: a sequential analysis of inflammatory mediators in respiratory fluids of high-risk preterm neonates. Pediatrics 1994;93:712718.

21. Thebaud B, Abman SH. Bronchopulmonary dysplasia: where have all the vessels gone? Roles of angiogenic growth factors in chronic lung disease. Am J Respir Crit Care Med 2007;175:978-985.

22. Galambos C, deMello DE. Molecular mechanisms of pulmonary vascular development. Pediatr Dev Pathol 2007;10:1-17.

23. Bogdanovic E, Nguyen VP, Dumont DJ. Activation of Tie2 by angiopoietin-1 and angiopoietin-2 results in their release and receptor internalization. J Cell Sci 2006;119:3551-3560.

24. Bose C, Van Marter LJ, Laughon M, O'Shea TM, Allred EN, Karna P, Ehrenkranz RA, Boggess K, Leviton A. Fetal growth restriction and chronic lung disease among infants born before the 28th week of gestation. Pediatrics 2009;124:e450-e458

25. Been JV, Zimmermann LJ. Histological chorioamnionitis and respiratory outcome in preterm infants. Arch Dis Child Fetal Neonatal Ed 2009;94:F218-225.

26. Laughon M, Allred EN, Bose C, O'Shea TM, Van Marter LJ, Ehrenkranz RA, Leviton A. Patterns of respiratory disease during the first 2 postnatal weeks in extremely premature infants. Pediatrics 2009;123:1124-1131. 
W. Thomas: Angiopoietin-2 in airways of ventilated preterm infants $\quad$ 19/22

1 27. Dammann O, Allred EN, Leviton A, Shen-Schwarz S, Heller D, Genest DR, Collins

2

3

4

MH. Fetal vasculitis in preterm newborns: interrelationships, modifiers, and antecedents. Placenta 2004;25:788-796.

28. Van Marter LJ, Dammann O, Allred EN, Leviton A, Pagano M, Moore M, Martin C. Chorioamnionitis, mechanical ventilation, and postnatal sepsis as modulators of chronic lung disease in preterm infants. J Pediatr 2002;140:171-176.

29. Speer CP. Chorioamnionitis, postnatal factors and proinflammatory response in the pathogenetic sequence of bronchopulmonary dysplasia. Neonatology 2009;95:353361.

30. Bhandari V, Choo-Wing R, Lee CG, Zhu Z, Nedrelow JH, Chupp GL, Zhang X, Matthay MA, Ware LB, Homer RJ, Lee PJ, Geick A, de Fougerolles AR, Elias JA. Hyperoxia causes angiopoietin 2-mediated acute lung injury and necrotic cell death. Nat Med 2006;12:1286-1293.

31. Karmpaliotis D, Kosmidou I, Ingenito EP, Hong K, Malhotra A, Sunday ME, Haley KJ. Angiogenic growth factors in the pathophysiology of a murine model of acute lung injury. Am J Physiol 2002;283:L585-595.

32. Thomas W, Seidenspinner S, Kramer BW, Kawczynska-Leda N, ChmielnickaKopaczyk M, Marx A, Wirbelauer J, Szymankiewicz M, Speer CP. Airway concentrations of angiopoietin-1 and endostatin in ventilated extremely premature infants are decreased after funisitis and unbalanced with bronchopulmonary dysplasia/death. Pediatr Res 2009;65:468-473.

33. Bhatt AJ, Pryhuber GS, Huyck H, Watkins RH, Metlay LA, Maniscalco WM. Disrupted pulmonary vasculature and decreased vascular endothelial growth factor, Flt-1, and TIE-2 in human infants dying with bronchopulmonary dysplasia. Am J Respir Crit Care Med 2001;164:1971-1980. 
W. Thomas: Angiopoietin-2 in airways of ventilated preterm infants $\quad 20 / 22$

1 34. Lassus P, Ristimaki A, Ylikorkala O, Viinikka L, Andersson S. Vascular endothelial

2

3

4 growth factor in human preterm lung. Am J Respir Crit Care Med 1999;159:14291433.

35. Been JV, Debeer A, van Iwaarden JF, Kloosterboer N, Passos VL, Naulaers G, Zimmermann LJ. Early alterations of growth factor patterns in bronchoalveolar lavage fluid from preterm infants developing bronchopulmonary dysplasia. Pediatr Res 2010;67:83-89.

36. Hansen AR, Barnes CM, Folkman J, McElrath TF. Maternal preeclampsia predicts the development of bronchopulmonary dysplasia. J Pediatr 2010;156:532-536.

37. Leinonen E, Wathen KA, Alfthan H, Ylikorkala O, Andersson S, Stenman UH, Vuorela P. Maternal serum angiopoietin-1 and -2 and tie-2 in early pregnancy ending in preeclampsia or intrauterine growth retardation. J Clin Endocrinol Metab 2010;95:126-133.

38. Bolin M, Wiberg-Itzel E, Wikstrom AK, Goop M, Larsson A, Olovsson M, Akerud H. Angiopoietin-1/angiopoietin-2 ratio for prediction of preeclampsia. Am J Hypertens 2009;22:891-895.

39. Buhimschi CS, Bhandari V, Dulay AT, Thung S, Razeq SS, Rosenberg V, Han CS, Ali UA, Zambrano E, Zhao G, Funai EF, Buhimschi IA. Amniotic fluid angiopoietin1, angiopoietin-2, and soluble receptor tunica interna endothelial cell kinase-2 levels and regulation in normal pregnancy and intraamniotic inflammation-induced preterm birth. J Clin Endocrinol Metab 2010;95:3428-3436.

40. Pacora P, Romero R, Chaiworapongsa T, Kusanovic JP, Erez O, Vaisbuch E, MazakiTovi S, Gotsch F, Jai Kim C, Than NG, Yeo L, Mittal P, Hassan SS. Amniotic fluid angiopoietin-2 in term and preterm parturition, and intra-amniotic infection/inflammation. J Perinat Med 2009;37:503-511. 
W. Thomas: Angiopoietin-2 in airways of ventilated preterm infants $\quad 21 / 22$

1 41. Thebaud B, Lacaze-Masmonteil T. If your placenta doesn't have it, chances are your 2 lungs don't have it either: the "vascular hypothesis" of bronchopulmonary dysplasia 3 starts in utero. J Pediatr 2010;156:521-523.

4 42. Reusch P, Barleon B, Weindel K, Martiny-Baron G, Godde A, Siemeister G, Marme 5 D. Identification of a soluble form of the angiopoietin receptor TIE-2 released from endothelial cells and present in human blood. Angiogenesis 2001;4:123-131.

7 43. Yuan HT, Khankin EV, Karumanchi SA, Parikh SM. Angiopoietin 2 is a partial 8 agonist/antagonist of Tie2 signaling in the endothelium. Mol Cell Biol 2009;29:20119 2022.

10 44. Fanaroff AA, Stoll BJ, Wright LL, Carlo WA, Ehrenkranz RA, Stark AR, Bauer CR, 11 Donovan EF, Korones SB, Laptook AR, Lemons JA, Oh W, Papile L-A, Shankaran S, 12 Stevenson DK, Tyson JE, Poole WK. Trends in neonatal morbidity and mortality for 13 very low birthweight infants. Am J Obstet Gynecol 2007;196:147.e141-147.e148. 
W. Thomas: Angiopoietin-2 in airways of ventilated preterm infants $\quad 22 / 22$

\section{Figure legends}

2

3 Figure 1: Boxplots of Ang2/SC (A) in TAF of the three groups control $(n=16)$,

4 chorioamnionitis $(n=9)$ and funisitis $(n=17)$. The bold bars indicate the median, the boxes the

5 interquartile range and the error bars the 5th and 95th percentile. Circles indicate outliers (1.5-

$63 \mathrm{x}$ interquartile range from upper or lower quartile), rhombs extreme values $(>3 \mathrm{x}$

7 interquartile range from upper or lower quartile). Ang2/SC in the funisitis group was

8 significantly lower than in the control group on days 1, 3 and 10 and lower than in the

9 chorioamnionitis group on days 1-10.

10

11 Figure 2: Boxplots of Ang2/SC in TAF for small for gestational age infants (SGA) compared 12 to appropriate for gestational age infants (AGA). SGA infants had significantly higher 13 Ang2/SC on days 1-7 than AGA infants ( $\mathrm{p}<0.05$, respectively)

15 Figure 3: Boxplots of the ratio Ang1/Ang2 in TAF for the outcome moderate BPD or death. 16 The ratio was significantly decreased on days 3-10 for infants who developed BPD or died.

18 Figure 4: Mean concentrations with standard deviations of Ang1, Ang2 and sTIE-2 in

19 umbilical cord serum of infants who survived without BPD and infants who developed BPD

20 or died . sTIE-2 concentrations were significantly higher for infants who developed BPD or 21 died. 
Table 1: Clinical characteristics and short term outcome

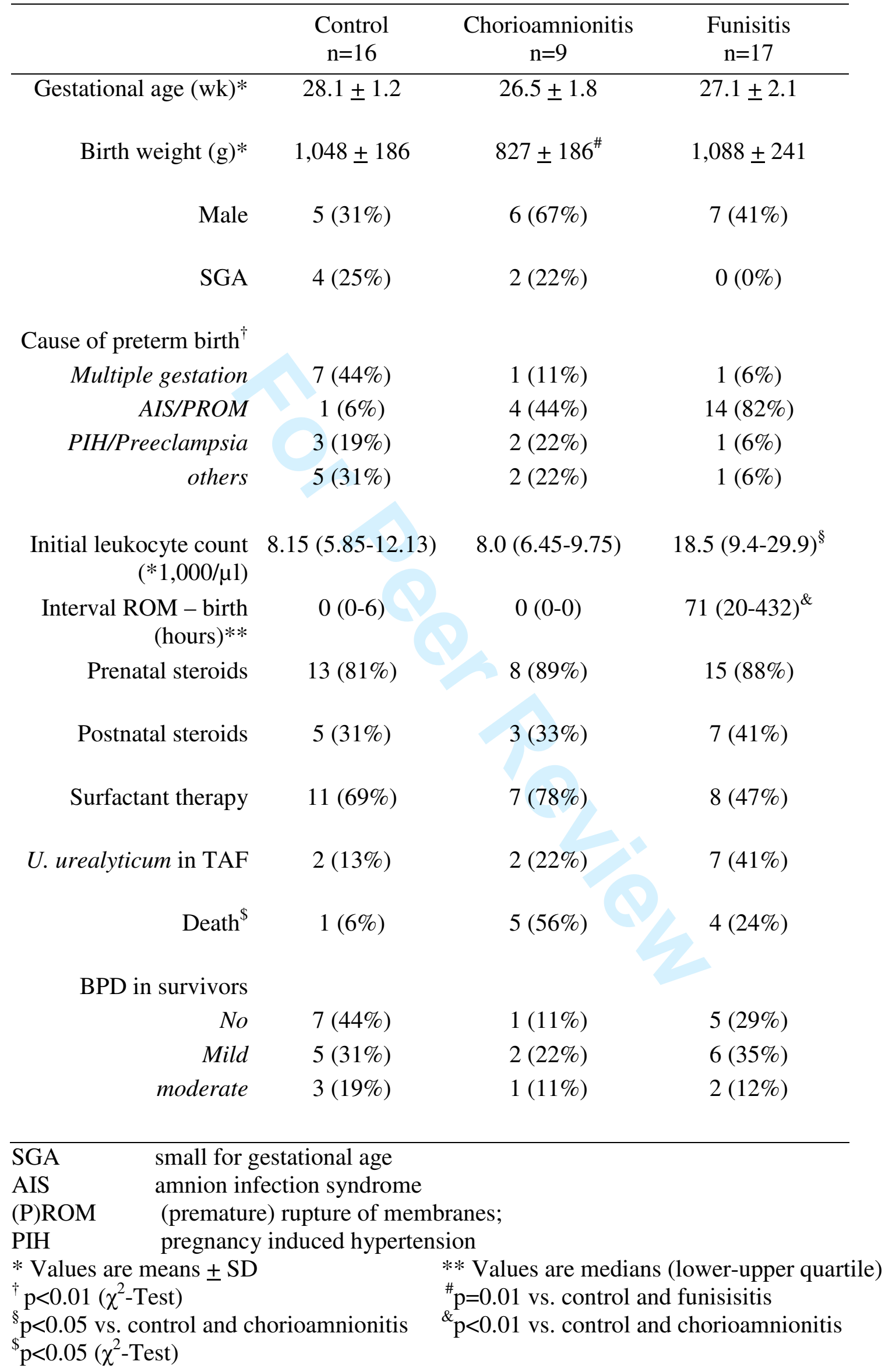


Table 2: Differences in clinical characteristics and respiratory support between infants who developed BPD or died and the remainder.

\begin{tabular}{|c|c|c|c|}
\hline & $\begin{array}{c}\text { BPD/death } \\
\mathrm{n}=29 \\
(\text { mean } \pm \mathrm{SD})\end{array}$ & $\begin{array}{l}\text { Survivors without BPD, } \\
\qquad \begin{array}{c}n=13 \\
(\text { mean } \pm \mathrm{SD})\end{array}\end{array}$ & $\mathrm{p}$-value \\
\hline Gestational age (wk) & $26.9 \pm 1.8$ & $28.3 \pm 1.4$ & $<0.05$ \\
\hline Birth weight (g) & $954 \pm 237$ & $1155 \pm 132$ & $<0.05$ \\
\hline $\begin{array}{l}\text { Duration of mechanical } \\
\text { ventilation (days) }\end{array}$ & $27.0 \pm 24.1$ & $13.3 \pm 5.1$ & $<0.01$ \\
\hline $\begin{array}{l}\text { Maximum peak oxygen } \\
\text { supplementation for }>0.5 \\
\text { hours }(\%)\end{array}$ & $85 \pm 21$ & $65 \pm 26$ & $<0.05$ \\
\hline $\begin{array}{l}\text { Maximum peak inspiratory } \\
\text { pressure }(\mathrm{kPa})\end{array}$ & $211 \pm 41$ & $181 \pm 27$ & $<0.05$ \\
\hline
\end{tabular}


Figure 1

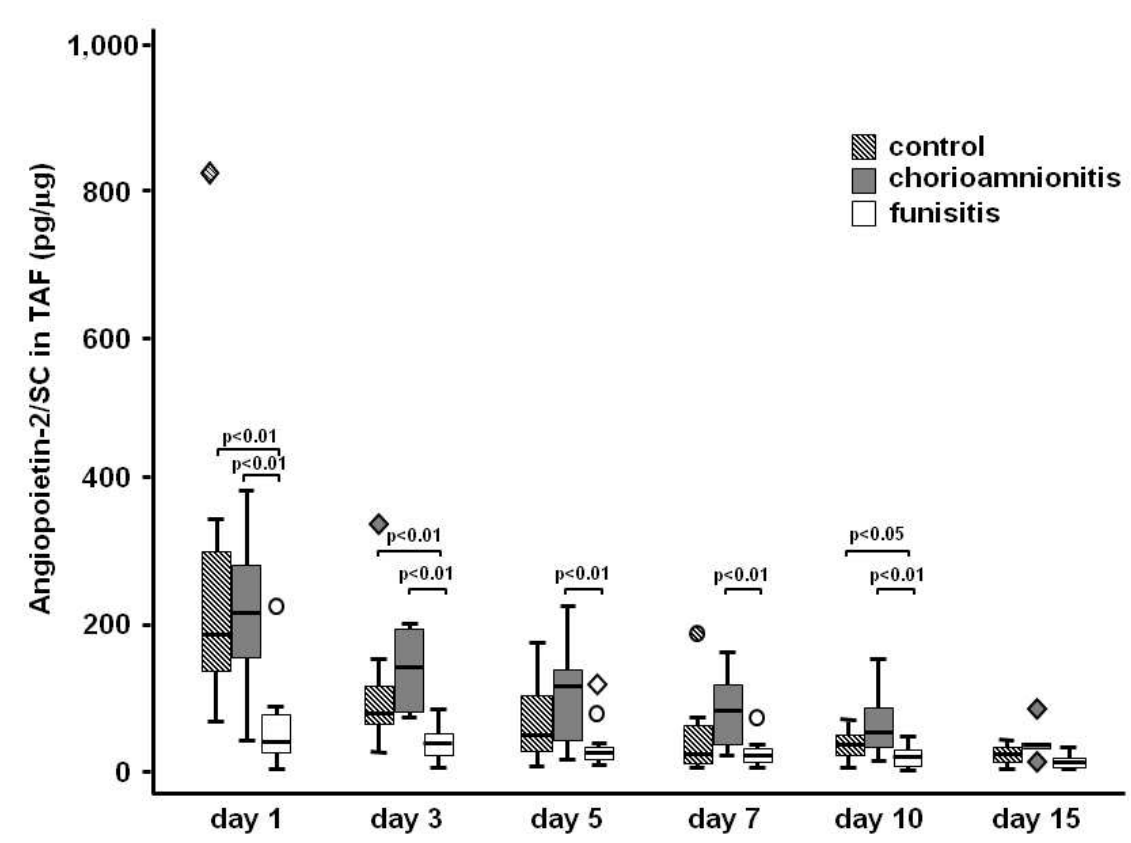

$254 \times 190 \mathrm{~mm}(96 \times 96 \mathrm{DPI})$

John Wiley \& Sons, Inc. 
Figure 2

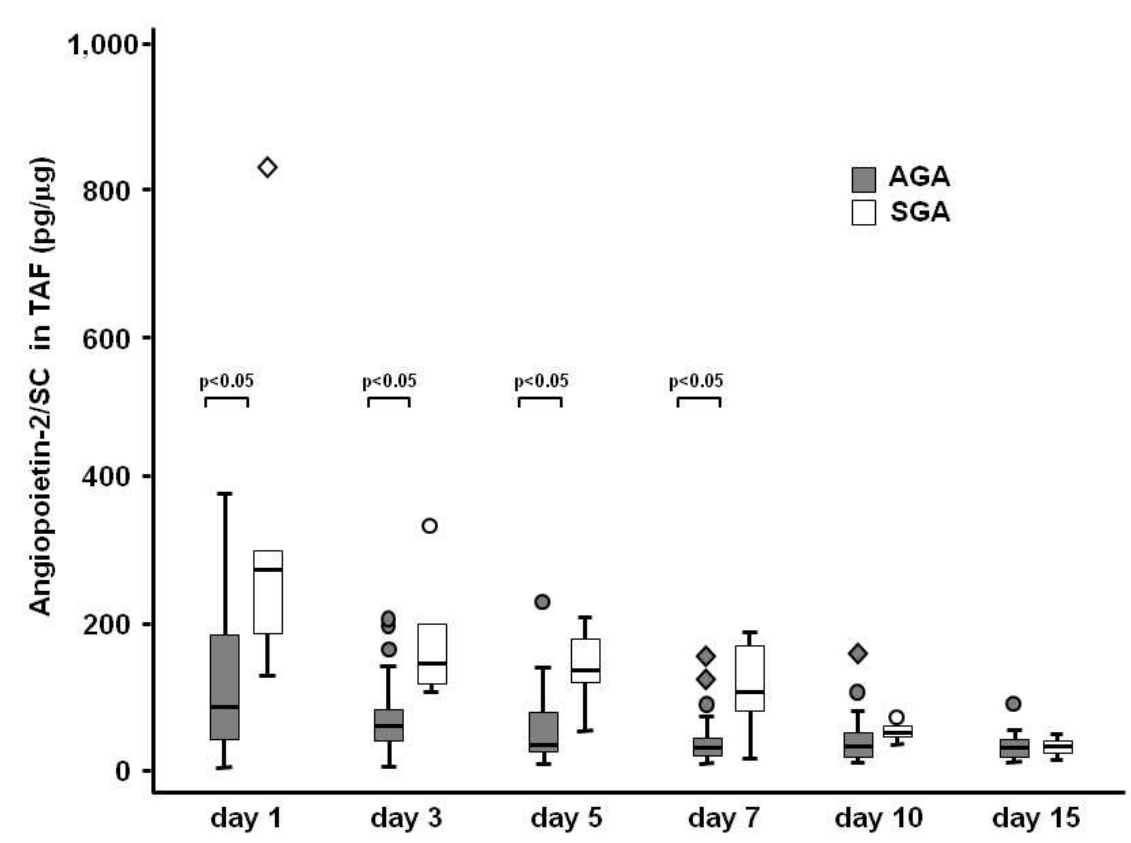

$254 \times 190 \mathrm{~mm}(96 \times 96$ DPI $)$

John Wiley \& Sons, Inc. 


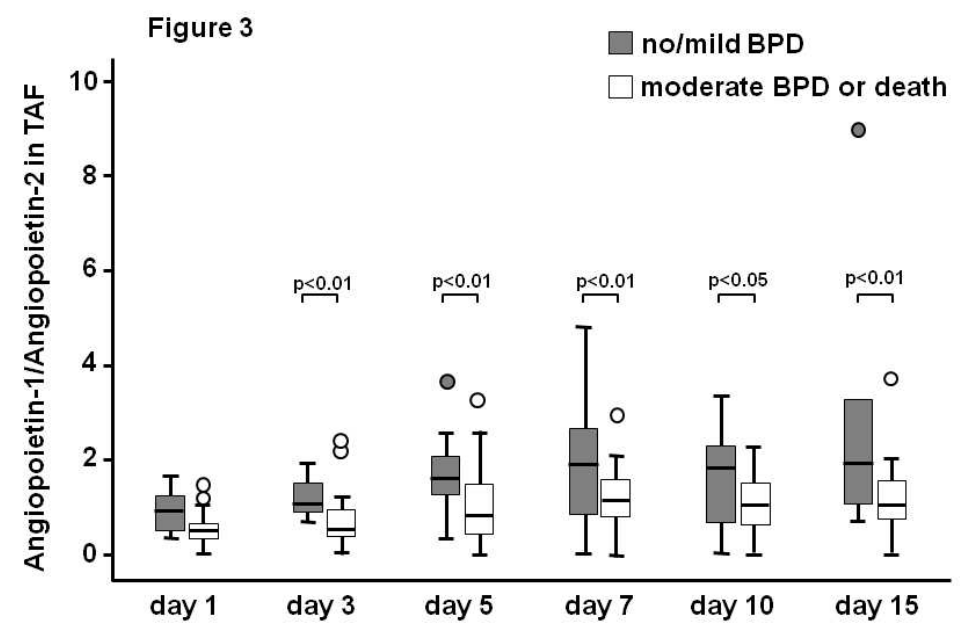

$296 \times 209 \mathrm{~mm}(96 \times 96$ DPI $)$

John Wiley \& Sons, Inc. 


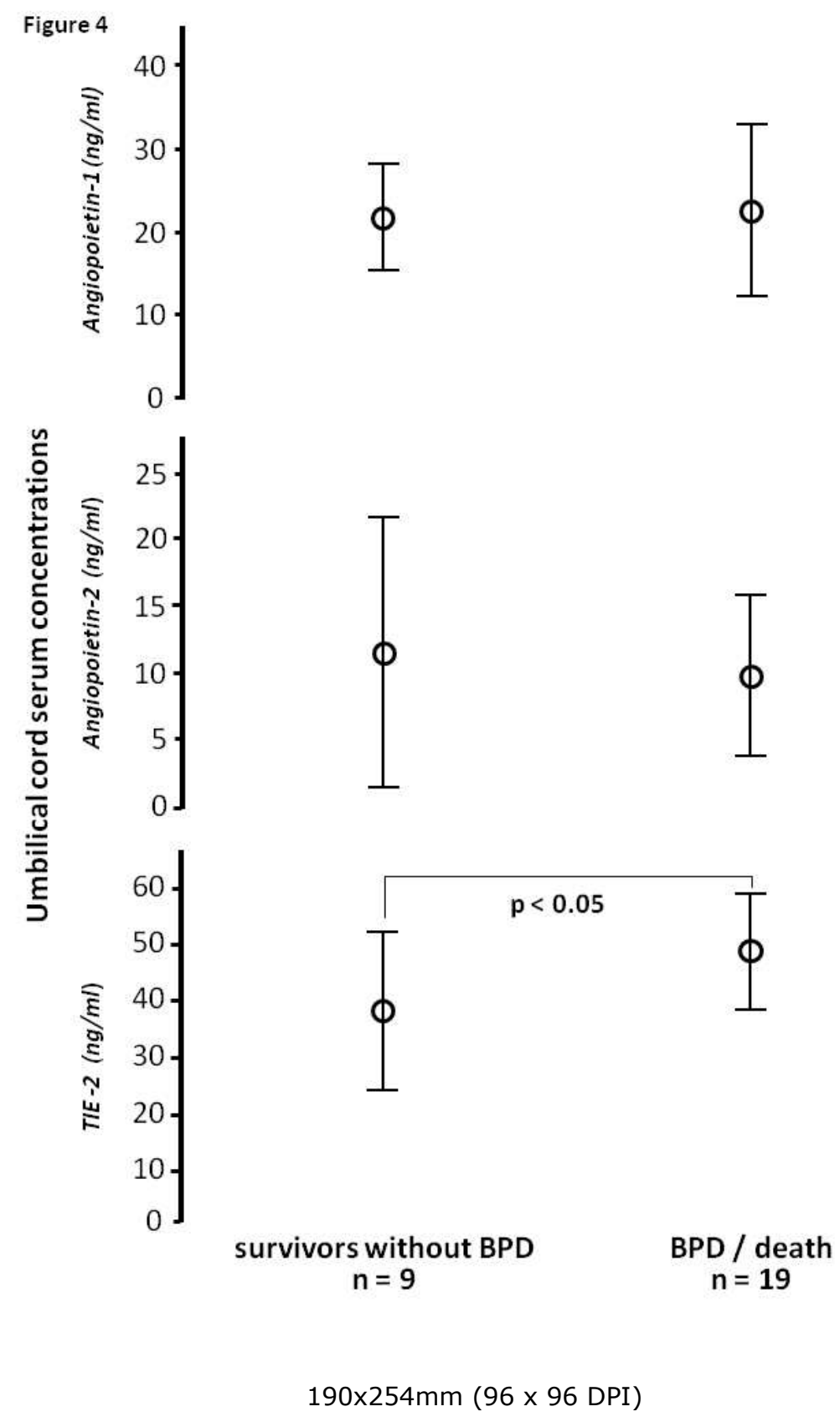

John Wiley \& Sons, Inc. 

3 4 5 6 7

1 Airway angiopoietin-2 in ventilated very preterm infants: association with prenatal factors and

2 neonatal outcome

3 Wolfgang Thomas ${ }^{1}$, Silvia Seidenspinner ${ }^{1}$, Boris W. Kramer ${ }^{2}$, Johannes Wirbelauer ${ }^{1}$, Natalia

4 Kawczyńska-Leda ${ }^{3}$, Marta Szymankiewicz ${ }^{3}$, Christian P. Speer ${ }^{1}$

$6 \quad{ }^{1}$ University Children's Hospital, University of Würzburg, Germany

$7 \quad{ }^{2}$ Department of Pediatrics, Maastricht University Medical Center, Maastricht, The

8 Netherlands

$9 \quad{ }^{3}$ Department of Neonatology, Poznań University of Medical Sciences, Poznań, Poland 10

12 This work was supported in part by a personal grant from G. Bierich, managing director,

13 Interfinanz, Duesseldorf, Germany

14 No author has competing interests to declare. 15

16 Key words: bronchopulmonary dysplasia, lung injury, angiogenesis,

17 vascular endothelial growth factor, small for gestational age infant 18

19 Corresponding author: Wolfgang Thomas, MD; University Children's Hospital, Josef-

20 Schneider-Str. 2, 97080 Würzburg, Germany, Tel.: +49(0)931-20127728,

21 Fax.: +49(0)931-20127714, e-mail: Thomas_w@klinik.uni-wuerzburg.de

22

23 Abbreviated title: Angiopoietin-2 in airways of ventilated preterm infants 
W. Thomas: Angiopoietin-2 in airways of ventilated preterm infants $\quad 2 / 22$

\section{Abstract}

2 Objective: Pulmonary angiogenesis is a prerequisite for lung development. Angiopoietin-2

3 (Ang2) destabilizes endothelial cells through its endothelial receptor TIE-2, enabling vascular

4 sprouting. Ang1 stabilizes new blood vessels. Soluble TIE-2 (sTIE-2) modulates these effects.

5 We hypothesized that histological funisitis is associated with alterations of Ang2 in airways

6 and of the systemic angiopoietin-TIE-2 homeostasis in very low birth weight infants

7 (VLBW), contributing to pulmonary morbidity and mortality.

8 Methods: We measured Ang2 in tracheobronchial aspirate fluid (TAF) of 42 VLBW $<30$

9 weeks of gestation from day 1 through 15 and Ang1, Ang2 and sTIE-2 in umbilical cord

10 serum of 28 infants by ELISA. Histological examination distinguished 3 groups: funisitis,

11 chorioamnionitis and controls.

12 Results: Funisitis was associated with lower Ang2 values in TAF but not with changes of

13 Ang1, Ang2 and sTIE-2 in umbilical cord serum. Infants who developed BPD or died had a

14 persistently decreased ratio of previously measured Ang1 to Ang2 in TAF on days 1-5 and

15 increased cord serum concentrations of sTIE-2. Moderate BPD/death was associated with an

16 increase of Ang2 in TAF on day 10 and decreased Ang1/Ang2 ratio from day 3-15. Small for

17 gestational age (SGA) infants had increased Ang2 in TAF on day 1-7 and a lower Ang1/Ang2

18 ratio on days $5-7$.

19 Conclusions: The predominance of Ang2 in airway fluid of infants with BPD/death and SGA

20 infants suggests a link between disrupted placental and fetal pulmonary angiogenesis.

21 Histological funisitis with reduced Ang2 in TAF was of minor relevance for outcome in our 22 cohort. 
W. Thomas: Angiopoietin-2 in airways of ventilated preterm infants $\quad 3 / 22$

\section{Introduction}

2 Angiogenesis plays a pivotal role in organ development and maturation ${ }^{1}$. In extremely

3 premature infants disturbed pulmonary angiogenesis accompanies alveolar simplification,

4 eventually leading to bronchopulmonary dysplasia (BPD) ${ }^{2-3}$.

5 Angiopoietin-2 (Ang2) belongs to a family of angiogenic growth factors which bind

6 specifically to the tyrosine kinase receptor with immunoglobulin and epidermal growth factor

7 homology domain 2 (TIE-2) on endothelial cells. Ang2 destabilizes the endothelium by

8 decreasing the phosphorylation status of TIE-2, leading to a more responsive endothelium, a

9 prerequisite for sprouting of new vessels ${ }^{4}$. The endothelial destabilization also leads to

10 vascular leakage which is a feature of inflammation ${ }^{5}$. Binding of the Ang2 antagonist

11 angiopoietin-1 (Ang1) to TIE-2 induces tightening of endothelial intercellular junctions,

12 leading to stabilization and maturation of newly formed vessels ${ }^{6}$. Both angiopoietins act

13 synergistically in the process of angiogenesis ${ }^{4}$. A soluble form of TIE-2 (sTIE-2), released

14 from the endothelium after proteolytical cleavage of the receptor's extracellular domain,

15 interferes with the angiopoietin-TIE-2 signaling by binding the free ligands ${ }^{7}$.

16 A differential spatial and temporal regulation of TIE-2 by Ang1 and Ang2 in the lung could

17 be shown a prerequisite for a stable pulmonary vasculature and normal lung development and 18 maturation in a murine model ${ }^{8}$. Increased concentrations of Ang2 in tracheobronchial aspirate

19 fluid (TAF) have been associated with the development of BPD or death in ventilated

20 premature infants ${ }^{9}$.

21 The role of angiopoietins and TIE- 2 in systemic inflammatory processes and pulmonary

22 injury has been extensively addressed. In a pediatric population, high plasma Ang2 levels and 23 low Ang1 levels were associated with septic shock ${ }^{10}$. Serum concentrations of sTIE-2 have 24 been shown elevated in adult septic patients at risk for adult respiratory distress syndrome 25 compared to non-septic patients ${ }^{11}$. However, data about blood levels of the angiopoietins and 26 their receptor in very premature infants exposed to prenatal inflammation do not exist. 
W. Thomas: Angiopoietin-2 in airways of ventilated preterm infants $\quad$ 4/22

1 Histological funisitis is regarded as morphological counterpart of a fetal systemic

2 inflammatory response $\mathrm{e}^{12}$ and has been shown associated with down-regulation of angiogenic

3 factors in placental tissue ${ }^{13}$. This fetal inflammatory response has been identified as risk factor

4 for $\mathrm{BPD}^{14}$ and mortality ${ }^{15}$ after premature birth.

5 The primary aim of our exploratory pilot study was to assess the association between

6 histological funisitis and postnatal airway concentrations of Ang2 in ventilated very

7 premature infants. We also investigated concentrations of the factors Ang1, Ang2 and sTIE-2

8 in umbilical cord blood. We speculated that alterations of pulmonary Ang2 and a disturbed

9 homeostasis of the angiopoietin-TIE-2 system after funisitis might contribute to pulmonary

10 morbidity and neonatal mortality of this high risk group of patients. 
W. Thomas: Angiopoietin-2 in airways of ventilated preterm infants $\quad 5 / 22$

\section{$1 \quad$ Material and Methods}

\section{Patients}

342 infants with a birth weight $<1500 \mathrm{~g}$ and a gestational age $<30$ weeks, who were admitted

4 to the intensive care unit of the Department of Neonatology, Poznań University of Medical

5 Sciences, Poland, between November 2004 and August 2005, were enrolled in this

6 prospective study after informed consent of the parents. Patients were inborns, had to be

7 mechanically ventilated for respiratory failure within the first 48 hours of life and had no

8 major congenital malformations. Respiratory support was adjusted to reach a $\mathrm{SpO}_{2}$ between

$985 \%$ and $94 \%$. Infants with supplemental oxygen for at least 28 days were assigned to have

$10 \mathrm{BPD}^{16}$. Depending on the need for oxygen or respiratory support at a postmenstrual age of 36

11 weeks, BPD was graded as mild, moderate or severe ${ }^{16}$. The study was approved by the Ethical

12 Committees of the Poznan University of Medical Sciences and the Faculty of Medicine,

13 University of Wuerzburg, Germany.

\section{Histological examinations}

16 For each infant at least 2 sections of umbilical cord, 2 sections of placental parenchyma and 1

17 section of membrane rolls were evaluated for the presence and severity of neutrophilic

18 infiltrates by two pathologists, unaware of clinical data, according to a protocol ${ }^{17}$. Patients

19 were assigned to three groups according to histological diagnosis. The first group was

20 characterized by chorioamnionitis and funisitis and will be referred to as funisitis group

21 ( $n=17)$. In the second group chorioamnionitis without funisitis was diagnosed

22 (chorioamnionitis group, $\mathrm{n}=9$ ). The control group consisted of 16 patients without histological 23 signs of inflammation in placenta, membranes and umbilical cord. 
W. Thomas: Angiopoietin-2 in airways of ventilated preterm infants $\quad 6 / 22$

\section{Sampling, preparation and storage}

2 Tracheobronchial aspiration was performed in a standardized manner up to 3 times per day on

3 days 1, 3, 5, 7, 10 and 15 of life. After instillation of $0.5 \mathrm{ml} \mathrm{NaCl} 0.9 \%$ into the endotracheal

4 tube a suction catheter was inserted slightly beyond the distal tip of the tube and airway

5 secretions were suctioned and collected in sterile mucus traps (Unomedical, Birkeroed,

6 Denmark). Each specimen was diluted with $\mathrm{NaCl} 0.9 \%$ to a volume of $0.5 \mathrm{ml}$. Specimens of

7 one day were pooled. They were spun at 3,000 revolutions per minute (rpm) for 5 minutes.

8 Supernatants were centrifuged again twice at 10,000 rpm for 5 minutes. Specimens were

9 cooled during processing, including centrifugation. The resulting clear supernatants

10 were divided into aliquots and stored at $-80^{\circ} \mathrm{C}$ until further analysis.

11 Umbilical cord blood could be collected from the umbilical cord vein of 28 infants. Blood 12 was centrifuged at $3,000 \mathrm{rpm}$ for 10 minutes and the serum stored at $-80^{\circ} \mathrm{C}$ until analysis.

14 Measurement of factors and secretory component for IgA

15 Concentrations of Ang1, Ang2 and sTIE-2 in umbilical cord serum and levels of Ang2 in 16 TAF were measured with commercially available enzyme-linked immunosorbent assays 17 (ELISA) (R\&D Systems, Minneapolis, USA). To correct for different dilutions, 18 concentrations in TAF were related to the secretory component for IgA (SC). SC levels in 19 TAF of preterm infants are largely independent of capillary leakage, gestational and postnatal 20 age as well as respiratory disease in the first weeks of life which makes it a valid dilutional 21 marker of epithelial lining fluid ${ }^{18-19}$. SC was measured by ELISA as previously described ${ }^{21}$.

23 Statistical analysis

24 Continuous variables were analyzed with one-way analysis of variance (ANOVA), if they 25 showed a normal distribution within groups and homogeneity of variance. Data with a skewed 
W. Thomas: Angiopoietin-2 in airways of ventilated preterm infants $\quad 7 / 22$

1 distribution were primarily assessed with nonparametric tests (Kruskal-Wallis test, Mann-

2 Whitney-U-test). We used post hoc Bonferroni method to correct for multiple comparisons.

3 Sequential values were compared with Friedman test and categorical or nominal values

4 assessed with $\chi^{2}$-test. Correlations were analyzed using Spearman's rank correlation

5 coefficients. All tests were two-sided, a value of $\mathrm{p}<0.05$ was considered significant. Statistical 6 evaluation was performed with SPSS 18.0 for Windows (SPSS Inc., Chicago, USA). 
W. Thomas: Angiopoietin-2 in airways of ventilated preterm infants $\quad 8 / 22$

\section{Results}

\section{Patients}

3 Clinical characteristics and outcome of patients in the 3 groups control, chorioamnionitis and

4 funisitis are summarized in table 1. Infants of the chorioamnionitis group had lower birth

5 weights than those in the control and funisitis group $(\mathrm{p}=0.01)$. Gestational age and proportions

6 of small for gestational age infants (SGA, i.e. birth weight $<10$. percentile for gestational age

7 and gender) did not differ significantly between groups. Most infants born after maternal

8 clinical signs of amnion infection and premature rupture of membranes belonged to the

9 funisitis group. The initial blood leukocyte count was higher and the interval between rupture

10 of membranes and birth was longer in the funisitis group compared to chorioamnionitis and

11 control $(\mathrm{p}<0.01$, respectively).

12 Thirty-six infants had received prenatal steroids. Within the first 15 days of life a single dose

13 of hydrocortisone was administered to 4 infants (days 1, 3, 6 and 9 respectively) and a single

14 dose of dexamethasone was given to 2 infants (day 8 and 10, respectively). Twenty-six infants

15 were treated with a natural surfactant preparation up to 3 times. Administration of prenatal

16 and postnatal corticosteroids or surfactant did not differ between groups.

17 There was no difference in respiratory outcome measures, such as duration of mechanical

18 ventilation, incidence of BPD or duration of oxygen therapy, between groups. TAF was

19 cultured for Ureaplasma urealyticum in 40 infants. Positive cultures (11) did not differ

20 between groups and were not associated with respiratory outcome either.

21 Mortality was highest in the chorioamnionitis group. Overall, 10 infants of the study cohort

22 died until day of life 25 . They had a significantly lower gestational age (mean \pm standard

23 deviation: $25.7 \pm 1.7$ weeks vs. $27.9 \pm 1.5$ weeks, $\mathrm{p}<0.01)$ and birth weight $(857 \pm 256 \mathrm{~g}$ vs.

$241067 \pm 199 \mathrm{~g}, \mathrm{p}<0.05)$ than survivors and were all mechanically ventilated from the day of

25 birth until death. 
W. Thomas: Angiopoietin-2 in airways of ventilated preterm infants

1 Nineteen infants developed BPD, 13 had mild, 6 moderate and no infant severe BPD. Infants,

2 who developed BPD or died, had a lower gestational age and birth weight and were more

3 likely to be males ( $\mathrm{p}<0.05$, respectively) than those who survived without BPD. They were

4 mechanically ventilated for a longer period $(\mathrm{p}<0.01)$ and needed higher peak oxygen

5 supplementation as well as higher peak inspiratory pressure on mechanical ventilation

6 ( $<<0.05$, respectively, table 2). All 6 SGA infants either developed BPD (5) or died (1).

8 Ang2/SC in $T A F$

9 Ang2/SC in TAF of the 42 infants decreased over time. Day 1, median: $110.4 \mathrm{pg} / \mu \mathrm{g}$

10 (interquartile range: 44.1-218.9); day 3, $71.8 \mathrm{pg} / \mu \mathrm{g}(44.3-106.6)$; day 5, $39.8 \mathrm{pg} / \mu \mathrm{g}(21.3-$

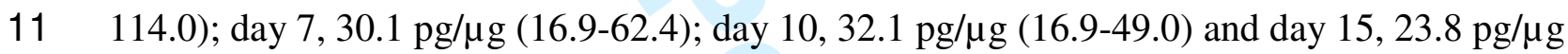

$12 \quad(7.7-35.6) ; \mathrm{p}<0.001$.

13 Figure 1 illustrates values for Ang2/SC in the 3 groups control, chorioamnionitis and funisitis.

14 The funisitis group had significantly lower values in TAF than the control group on days 1-3

$15(\mathrm{p}<0.01)$ and $10(\mathrm{p}<0.05)$ and lower values than the chorioamnionitis group on days 1-7 $(\mathrm{p}<$

16 0.01, respectively), whereas Ang2/SC did not differ significantly between the

17 chorioamnionitis and control group on any day.

18 Infants who developed a moderate form of BPD or died showed significantly higher Ang2/SC

19 on day $10(49.5 \mathrm{pg} / \mu \mathrm{g}$ [38.2-72.5]) compared to the remainder (24.2 pg/ $\mu \mathrm{g}$ [15.8-36.5], p

$20<0.05)$.

21 Ang2/SC did not correlate with gestational age and did not differ between genders. On days

22 1-7 SGA infants had significantly higher Ang2/SC levels than those infants whose birth

23 weight was appropriate for gestational age (AGA) (figure 2). Surfactant therapy, pre- and

24 postnatal corticosteroids and colonization with Ureaplasma urealyticum were not associated

25 with alterations of Ang2/SC. 
W. Thomas: Angiopoietin-2 in airways of ventilated preterm infants $\quad 10 / 22$

\section{$1 \quad$ Ratio Ang1/Ang2}

2 We used TAF concentrations of the Ang2 counterpart Ang1, which we had previously

3 measured in the same cohort, to calculate the ratio between the two factors ${ }^{32}$. The Ang1/Ang2

4 ratio did not differ significantly between the three groups control, chorioamnionitis and

5 funisitis (data not shown).

6 On days 1-5, the ratio was significantly lower in infants who developed BPD or died $(\mathrm{p}<0.05$,

7 respectively). Patients, who developed moderate BPD or died, had significantly lower values

8 on days 3-15 compared to the remaining infants ( $\mathrm{p}<0.05$, respectively) (figure 3 ). The ratio

9 was significantly decreased in SGA infants compared to AGA infants on days 5 and 7 (p<

$100.05)$.

11 Umbilical cord serum concentrations

12 Umbilical cord blood could be obtained from 28 infants of our cohort. They did not differ

13 significantly in any demographic or outcome parameter from the remaining 14 infants.

14 Umbilical cord serum concentrations of Ang1, Ang2 and sTie-2 were not different between

15 the 3 groups control, chorioamnionitis and funisitis. They did not differ between SGA and

16 AGA infants either. Patients, who developed BPD or died, had significantly higher sTIE-2

17 concentrations $(\mathrm{p}<0.05)$ than those that survived without BPD (figure 4). 
W. Thomas: Angiopoietin-2 in airways of ventilated preterm infants $\quad$ 11/22

\section{Discussion}

2 In our exploratory study histological funisitis was associated with decreased concentrations of

3 Ang2 in TAF of ventilated very premature infants compared to infants exposed to

4 chorioamnionitis or to no inflammation in utero. Pulmonary angiogenesis is a prerequisite for

5 alveolarisation ${ }^{2,21}$ and Ang2 is responsible for endothelial cell sprouting and promotes

6 vascular remodeling ${ }^{22}$. In vitro, proangiogenic signaling of Ang2 by activation of TIE-2 has

7 been shown concentration dependent ${ }^{23}$. One might therefore speculate that decreased

8 proangiogenic signaling by Ang2 in the lungs of very premature infants with funisitis -

9 reflecting a fetal systemic inflammatory response ${ }^{12}$ - may predispose them for disturbed

10 pulmonary angiogenesis along with impaired alveolar development, eventually contributing to

11 the risk of $\mathrm{BPD}^{14-15}$.

12 However, we found higher Ang2 concentrations in TAF of ventilated VLBW infants on day

1310 to be associated with the outcome moderate BPD or death along with a reduced ratio of

14 Ang1 to Ang2 in TAF on many of the first postnatal days in infants who developed mild to

15 moderate BPD or died. These findings suggest that a preponderance of Ang2 in airway fluid

16 of ventilated very premature infants plays a role for pulmonary outcome and neonatal

17 mortality. Increased Ang2 concentrations in TAF in the first week of life of ventilated VLBW

18 infants have recently been shown associated with the combined outcome moderate BPD or

19 death $^{9}$. Our findings confirm and amend these data.

20 In recent years, the association between histological funisitis and respiratory morbidity in very

21 premature infants has been questioned ${ }^{24-26}$. In our cohort funisitis was not associated with the

22 outcome BPD or death. This may be due to a lack of power to detect such an association with

23 our samples size. Moreover, funisitis does not represent a homogeneous disease ${ }^{27}$. Its risk is

24 modified by factors, like duration of membrane rupture, which by themselves interfere with

25 neonatal outcome. Need for sustained mechanical ventilation and higher oxygen 
W. Thomas: Angiopoietin-2 in airways of ventilated preterm infants

$12 / 22$

1 between prenatal inflammation and BPD ${ }^{26,28-29}$, were both associated with the combined

2 outcome BPD or death in our cohort. Hyperoxia has been shown to induce pulmonary Ang2

3 expression on transcriptional and translational level in an animal model of acute lung injury

4 and to decrease Ang1 mRNA expression simultaneously ${ }^{30}$. In this setting the abundance of

5 Ang2 seems to play an important role for pulmonary oxidative injury, inflammation and cell

6 death $^{30}$. These experimental data are also in accordance with our findings and underscore the

7 relevance of postnatal factors as potential risk modulators for $\mathrm{BPD}^{28}$.

8 Treatment strategies targeting to reduce pulmonary Ang2 expression may have potential

9 benefits for respiratory outcome ${ }^{30}$. Ang2 concentrations in TAF of VLBW infants have been

10 decreased after administration of dexamethasone ${ }^{9}$. However, our data suggest that the overall

11 balance between different types of factors - i.e. between Ang1, which stabilizes endothelium

12 and leads to vascular maturation ${ }^{6}$, and Ang2, which destabilizes vascular endothelium ${ }^{4-5}$ - may

13 be as important for respiratory outcome of very premature infants as changes in one single

14 factor. This is in accordance with data from a model of lipopolysaccharide-induced acute lung

15 injury $^{31}$. Likewise, we have recently demonstrated an imbalance between Ang1 and the

16 angiostatic factor endostatin in TAF of extremely premature infants to be associated with the

17 combined outcome BPD or death ${ }^{32}$.

18 The functions of angiopoietins are pleiotropic and context-dependent ${ }^{1}$. Ang2 synergizes with 19 vascular endothelial growth factor (VEGF) to stimulate angiogenesis but causes endothelial 20 cell death and vessel regression in the absence of sufficient angiogenic signals ${ }^{1}$. VEGF has 21 been shown decreased in TAF of ventilated very premature infants developing BPD and in 22 pulmonary tissue of infants who had died from the disease ${ }^{33-35}$. In light of these data our 23 findings would support the concept of impaired vascular signaling in the pathogenesis of 24 BPD.

25 Interestingly, Ang2 levels in TAF of SGA infants were increased compared to AGA infant 26 during the first week of life in our cohort, along with an imbalance between Ang1 and Ang2 
W. Thomas: Angiopoietin-2 in airways of ventilated preterm infants 13/22

1 on day 5 and 7. All SGA infants either developed BPD or died. These findings are in line with

22 recent studies, which identified preeclampsia and intrauterine growth retardation (IUGR),

3 but not histological chorioamnionitis with funisitis, as risk factors for BPD in extremely

4 premature infants ${ }^{24,36}$.

5 Regulation of vascular development in the feto-maternal unit is disturbed in preeclampsia,

6 leading to disturbed placentation and eventually to IUGR. Similarly, disrupted pulmonary

7 vascularisation plays a crucial role for $\mathrm{BPD}^{2,3,21}$. Our data complement existing evidence that

8 an imbalance between Ang1 and Ang2 in different feto-maternal compartments is a common

9 denominator of impaired placental as well as fetal pulmonary vascular development. In

10 maternal plasma at midgestation, IUGR has been found associated with increased Ang2

11 concentrations $^{37}$ and preeclampsia with a decrease of the Ang1/Ang2 ratio $^{38}$. A decrease of

12 Ang2 concentrations along with an increase of the Ang1/Ang2 ratio in the amniotic cavity

13 seems to be critical to prevent regression, destabilization and leakage of placental vessels

14 during normal gestation ${ }^{39-40}$. Intraamniotic Ang2 might be at least partially of fetal pulmonary

15 origin $^{30,39}$. Higher Ang2 level in TAF during the initial phase of life in SGA infants might

16 therefore reflect both disturbed placental and fetal pulmonary angiogenesis ${ }^{41}$.

17 Remarkably, intraamniotic inflammation/infection - assessed by either the presence of soluble

18 markers of the innate immune system, increased intraamniotic IL-6 or positive amniotic fluid

19 cultures - have been shown associated with higher intraamniotic Ang2 levels in women

20 presenting with preterm labor or preterm premature rupture of membranes in 2 studies ${ }^{39-40}$.

21 Both studies failed to demonstrate an association between histological funisitis and higher

22 intraamniotic Ang2. In placentas of very premature infants angiogenic factors have been

23 demonstrated to be decreased after histological chorioamnionitis and funisitis ${ }^{13}$. However,

24 histological evidence of inflammation was not associated with morphological alterations of

25 the placental vasculature in this study. These data underscore a limited relevance of

26 histological chorioamionitis with funisitis for angiogenic homeostasis in maternal and fetal 
W. Thomas: Angiopoietin-2 in airways of ventilated preterm infants

1 tissues. Likewise, histological funisitis was not associated with changes of umbilical cord

2 serum concentrations of Ang1, Ang2 and sTIE-2 concentrations in our cohort.

3 However, sTIE-2 levels in umbilical cord serum were higher in infants who developed BPD

4 or died. In critically ill adult patients concentrations of sTIE-2 in blood have been associated

5 with increased pulmonary vascular permeability ${ }^{11}$, which plays a role in the early phase of

$6 \mathrm{BPD}^{16,20,29}$. sTIE-2 is released into the blood after proteolytical cleavage of the extracellular

7 domain of the endothelial receptor, either constitutively or after proangiogenic stimulation ${ }^{7,42}$.

8 sTIE-2 is believed to act as a decoy for angiopoietins, affecting the signaling pathways

9 induced by binding of the ligands to the membrane-bound receptor ${ }^{42}$. As Ang1 has been

10 shown to bind to sTIE-2 with a much higher affinity than Ang2 in vitro ${ }^{43}$, high sTIE-2

11 concentrations may shift the Ang1/Ang2 ratio in favour of Ang2, eventually contributing to

12 systemic and pulmonary endothelial activation and destabilization ${ }^{11}$.

13 Lower gestational and birth weight as well as sustained mechanical ventilation of infants who

14 died in our cohort represent important risk factors for all grades of BPD. We therefore

15 combined the outcome BPD and death for analysis. The higher mortality in the

16 chorioamnionitis group compared to both other groups can be attributed to the lower birth

17 weight in this group as important predictor of mortality in VLBW infants ${ }^{44}$.

18 Our clinical explorative study has limitations. We did not assess the activity but only

19 concentrations of factors involved in angiogenesis. Moreover, our sample size made inclusion

20 of potential confounders in a multivariate analysis not applicable as the power to detect

21 associations between independent factors and concentrations of the angiogenic factors would

22 have been less than $80 \%$ in most cases. Therefore, our study can only be regarded preliminary

23 and suitable to generate new hypotheses, which have to be proved by experimental studies

24 and clinical studies with appropriate patient numbers to allow multivariate comparisons,

25 before definite conclusions can be drawn. 
W. Thomas: Angiopoietin-2 in airways of ventilated preterm infants $\quad 15 / 22$

1 To conclude, our data suggest that an increase of Ang2 and an imbalance with its counterpart

2 Ang1 in airway fluid potentially contributes to a sustained disturbance of pulmonary vascular

3 and alveolar development in ventilated very preterm infants who develop BPD or die. The

4 presence of such an imbalance in SGA infants implies an association between disrupted

5 placental and fetal pulmonary angiogenesis. We demonstrated that histological funisitis

6 associated with reduced Ang2 concentrations in airway fluid is of minor relevance for

7 outcome. Further ante- and postnatal factors are likely to modulate the risk of disturbed

8 pulmonary vascularisation and alveolarisation in very premature infants.

9

10 Acknowledgement

11 We thank Alexander Marx, Institute of Pathology, University Hospital Mannheim, Germany 12 and Maria Chmielnicka-Kopaczyk, Department of Pathology, Poznań University of Medical

13 Sciences, Poznań, Poland for histological evaluation and Daniela Keller, Department of 14 Bioinformatics, University of Wuerzburg, Germany, for statistical advice.

17 No author has competing interests to declare. 
W. Thomas: Angiopoietin-2 in airways of ventilated preterm infants $\quad 16 / 22$

1 References

2

3 1. Carmeliet P. Angiogenesis in health and disease. Nature Med 2003;9:653-660.

42 2. Thebaud B. Angiogenesis in lung development, injury and repair: implications for

$5 \quad$ chronic lung disease of prematurity. Neonatology 2007;9:291-297.

6 3. Stenmark KR, Abman SH. Lung vascular development: implications for the pathogenesis of bronchopulmonary dysplasia. Ann Rev Physiol 2005;67:623-661.

8 4. Distler JH, Hirth A, Kurowska-Stolarska M, Gay RE, Gay S, Distler O. Angiogenic and angiostatic factors in the molecular control of angiogenesis. Q J Nucl Med 2003;47:149-161.

11 5. Roviezzo F, Tsigkos S, Kotanidou A, Bucci M, Brancaleone V, Cirino G,

Papapetropoulos A. Angiopoietin-2 causes inflammation in vivo by promoting vascular leakage. J Pharmacol Exp Ther 2005;314:738-744.

6. Makinde T, Agrawal DK. Intra and extravascular transmembrane signalling of angiopoietin-1-Tie2 receptor in health and disease. J Cell Mol Med 2008;12:810-828.

7. Findley CM, Cudmore MJ, Ahmed A, Kontos CD. VEGF Induces Tie2 shedding via a phosphoinositide 3-kinase/Akt dependent pathway to modulate Tie2 signaling. Arterioscler Thromb Vasc Biol 2007;27:2619-2626.

19 8. Hato T, Kimura Y, Morisada T, Koh GY, Miyata K, Tabata M, Kadomatsu T, Endo M, Urano T, Arai F, Araki K, Suda T, Kobayashi K, Oike Y. Angiopoietins contribute

23 9. Aghai ZH, Faqiri S, Saslow JG, Nakhla T, Farhath S, Kumar A, Eydelman R, Strande L, Stahl G, Leone P, Bhandari V. Angiopoietin 2 concentrations in infants developing to lung development by regulating pulmonary vascular network formation. Biochem Biophys Res Commun 2009;381:218-223. bronchopulmonary dysplasia: attenuation by dexamethasone. J Perinatol 2008;28:149155. 
W. Thomas: Angiopoietin-2 in airways of ventilated preterm infants $\quad$ 17/22

1 10. Giuliano JS, Jr., Lahni PM, Harmon K, Wong HR, Doughty LA, Carcillo JA,

2

3

4

Zingarelli B, Sukhatme VP, Parikh SM, Wheeler DS. Admission angiopoietin levels in children with septic shock. Shock 2007;28:650-654.

11. van der Heijden M, van Nieuw Amerongen GP, van Hinsbergh VW, Groeneveld AJ. The interaction of soluble Tie2 with angiopoietins and pulmonary vascular permeability in septic and non-septic critically ill patients. Shock 2010;33:263-268.

12. Pacora P, Chaiworapongsa T, Maymon E, Kim YM, Gomez R, Yoon BH, Ghezzi F, Berry SM, Qureshi F, Jacques SM, Kim JC, Kadar N, Romero R. Funisitis and chorionic vasculitis: the histological counterpart of the fetal inflammatory response syndrome. J Matern Fetal Neonatal Med 2002;11:18-25.

13. Kramer BW, Kaemmerer U, Kapp M, Herbst D, Marx A, Berg D, Groneck PA, Speer CP. Decreased expression of angiogenic factors in placentas with chorioamnionitis after preterm birth. Pediatr Res 2005;58:607-612.

14. Yoon BH, Romero R, Kim KS, Park JS, Ki SH, Kim BI, Jun JK. A systemic fetal inflammatory response and the development of bronchopulmonary dysplasia. Am J Obstet Gynecol 1999;181:773-779.

15. Lau J, Magee F, Qiu Z, Hoube J, Von Dadelszen P, Lee SK. Chorioamnionitis with a fetal inflammatory response is associated with higher neonatal mortality, morbidity, and resource use than chorioamnionitis displaying a maternal inflammatory response only. Am J Obstet Gynecol 2005;193:708-713.

16. Jobe AH, Bancalari E. Bronchopulmonary dysplasia. Am J Respir Crit Care Med 2001;163(7):1723-1729.

17. Rogers BB, Alexander JM, Head J, McIntire D, Leveno KJ. Umbilical vein interleukin-6 levels correlate with the severity of placental inflammation and gestational age. Hum Pathol 2002;33:335-340. 
W. Thomas: Angiopoietin-2 in airways of ventilated preterm infants $\quad 18 / 22$

1 18. Dargaville PA, South M, Vervaart P, McDougall PN. Validity of markers of dilution

2

3

4

5

6

7

8

9

10

11

12

13

14

15

16

17

18

19

20

21

22

23

24 in small volume lung lavage. Am J Respir Crit Care Med 1999;16:778-784.

19. Watts CL, Bruce MC. Comparison of secretory component for immunoglobulin A with albumin as reference proteins in tracheal aspirate from preterm infants. J Pediatr 1995; 127:113-122.

20. Groneck P, Götze-Speer B, Oppermann M, Eiffert H, Speer CP. Association of pulmonary inflammation and increased microvascular permeability during the development of bronchopulmonary dysplasia: a sequential analysis of inflammatory mediators in respiratory fluids of high-risk preterm neonates. Pediatrics 1994;93:712718.

21. Thebaud B, Abman SH. Bronchopulmonary dysplasia: where have all the vessels gone? Roles of angiogenic growth factors in chronic lung disease. Am J Respir Crit Care Med 2007;175:978-985.

22. Galambos C, deMello DE. Molecular mechanisms of pulmonary vascular development. Pediatr Dev Pathol 2007;10:1-17.

23. Bogdanovic E, Nguyen VP, Dumont DJ. Activation of Tie2 by angiopoietin-1 and angiopoietin-2 results in their release and receptor internalization. J Cell Sci 2006;119:3551-3560.

24. Bose C, Van Marter LJ, Laughon M, O'Shea TM, Allred EN, Karna P, Ehrenkranz RA, Boggess K, Leviton A. Fetal growth restriction and chronic lung disease among infants born before the 28th week of gestation. Pediatrics 2009;124:e450-e458

25. Been JV, Zimmermann LJ. Histological chorioamnionitis and respiratory outcome in preterm infants. Arch Dis Child Fetal Neonatal Ed 2009;94:F218-225.

26. Laughon M, Allred EN, Bose C, O'Shea TM, Van Marter LJ, Ehrenkranz RA, Leviton A. Patterns of respiratory disease during the first 2 postnatal weeks in extremely premature infants. Pediatrics 2009;123:1124-1131. 
W. Thomas: Angiopoietin-2 in airways of ventilated preterm infants

$19 / 22$

27. Dammann O, Allred EN, Leviton A, Shen-Schwarz S, Heller D, Genest DR, Collins

2

3 MH. Fetal vasculitis in preterm newborns: interrelationships, modifiers, and antecedents. Placenta 2004;25:788-796.

28. Van Marter LJ, Dammann O, Allred EN, Leviton A, Pagano M, Moore M, Martin C. Chorioamnionitis, mechanical ventilation, and postnatal sepsis as modulators of chronic lung disease in preterm infants. J Pediatr 2002;140:171-176.

29. Speer CP. Chorioamnionitis, postnatal factors and proinflammatory response in the pathogenetic sequence of bronchopulmonary dysplasia. Neonatology 2009;95:353361.

30. Bhandari V, Choo-Wing R, Lee CG, Zhu Z, Nedrelow JH, Chupp GL, Zhang X, Matthay MA, Ware LB, Homer RJ, Lee PJ, Geick A, de Fougerolles AR, Elias JA. Hyperoxia causes angiopoietin 2-mediated acute lung injury and necrotic cell death. Nat Med 2006;12:1286-1293.

31. Karmpaliotis D, Kosmidou I, Ingenito EP, Hong K, Malhotra A, Sunday ME, Haley KJ. Angiogenic growth factors in the pathophysiology of a murine model of acute lung injury. Am J Physiol 2002;283:L585-595.

32. Thomas W, Seidenspinner S, Kramer BW, Kawczynska-Leda N, ChmielnickaKopaczyk M, Marx A, Wirbelauer J, Szymankiewicz M, Speer CP. Airway concentrations of angiopoietin- 1 and endostatin in ventilated extremely premature infants are decreased after funisitis and unbalanced with bronchopulmonary dysplasia/death. Pediatr Res 2009;65:468-473.

33. Bhatt AJ, Pryhuber GS, Huyck H, Watkins RH, Metlay LA, Maniscalco WM. Disrupted pulmonary vasculature and decreased vascular endothelial growth factor, Flt-1, and TIE-2 in human infants dying with bronchopulmonary dysplasia. Am J Respir Crit Care Med 2001;164:1971-1980. 
W. Thomas: Angiopoietin-2 in airways of ventilated preterm infants $\quad 20 / 22$

1 34. Lassus P, Ristimaki A, Ylikorkala O, Viinikka L, Andersson S. Vascular endothelial

2

3

4 growth factor in human preterm lung. Am J Respir Crit Care Med 1999;159:14291433.

35. Been JV, Debeer A, van Iwaarden JF, Kloosterboer N, Passos VL, Naulaers G, Zimmermann LJ. Early alterations of growth factor patterns in bronchoalveolar lavage fluid from preterm infants developing bronchopulmonary dysplasia. Pediatr Res 2010;67:83-89.

36. Hansen AR, Barnes CM, Folkman J, McElrath TF. Maternal preeclampsia predicts the development of bronchopulmonary dysplasia. J Pediatr 2010;156:532-536.

37. Leinonen E, Wathen KA, Alfthan H, Ylikorkala O, Andersson S, Stenman UH, Vuorela P. Maternal serum angiopoietin-1 and -2 and tie-2 in early pregnancy ending in preeclampsia or intrauterine growth retardation. J Clin Endocrinol Metab 2010;95:126-133.

38. Bolin M, Wiberg-Itzel E, Wikstrom AK, Goop M, Larsson A, Olovsson M, Akerud H. Angiopoietin-1/angiopoietin-2 ratio for prediction of preeclampsia. Am J Hypertens 2009;22:891-895.

39. Buhimschi CS, Bhandari V, Dulay AT, Thung S, Razeq SS, Rosenberg V, Han CS, Ali UA, Zambrano E, Zhao G, Funai EF, Buhimschi IA. Amniotic fluid angiopoietin1, angiopoietin-2, and soluble receptor tunica interna endothelial cell kinase-2 levels and regulation in normal pregnancy and intraamniotic inflammation-induced preterm birth. J Clin Endocrinol Metab 2010;95:3428-3436.

40. Pacora P, Romero R, Chaiworapongsa T, Kusanovic JP, Erez O, Vaisbuch E, MazakiTovi S, Gotsch F, Jai Kim C, Than NG, Yeo L, Mittal P, Hassan SS. Amniotic fluid angiopoietin-2 in term and preterm parturition, and intra-amniotic infection/inflammation. J Perinat Med 2009;37:503-511. 
W. Thomas: Angiopoietin-2 in airways of ventilated preterm infants $\quad 21 / 22$

1 41. Thebaud B, Lacaze-Masmonteil T. If your placenta doesn't have it, chances are your 2 lungs don't have it either: the "vascular hypothesis" of bronchopulmonary dysplasia $3 \quad$ starts in utero. J Pediatr 2010;156:521-523.

4 42. Reusch P, Barleon B, Weindel K, Martiny-Baron G, Godde A, Siemeister G, Marme 5 D. Identification of a soluble form of the angiopoietin receptor TIE-2 released from endothelial cells and present in human blood. Angiogenesis 2001;4:123-131.

7 43. Yuan HT, Khankin EV, Karumanchi SA, Parikh SM. Angiopoietin 2 is a partial 8 agonist/antagonist of Tie2 signaling in the endothelium. Mol Cell Biol 2009;29:20119 2022.

10 44. Fanaroff AA, Stoll BJ, Wright LL, Carlo WA, Ehrenkranz RA, Stark AR, Bauer CR, 11 Donovan EF, Korones SB, Laptook AR, Lemons JA, Oh W, Papile L-A, Shankaran S, 12 Stevenson DK, Tyson JE, Poole WK. Trends in neonatal morbidity and mortality for 13 very low birthweight infants. Am J Obstet Gynecol 2007;196:147.e141-147.e148. 
W. Thomas: Angiopoietin-2 in airways of ventilated preterm infants $\quad 22 / 22$

\section{Figure legends}

2

3 Figure 1: Boxplots of Ang2/SC (A) in TAF of the three groups control $(n=16)$,

4 chorioamnionitis $(n=9)$ and funisitis $(n=17)$. The bold bars indicate the median, the boxes the

5 interquartile range and the error bars the 5th and 95th percentile. Circles indicate outliers (1.5-

$63 \mathrm{x}$ interquartile range from upper or lower quartile), rhombs extreme values $(>3 \mathrm{x}$

7 interquartile range from upper or lower quartile). Ang2/SC in the funisitis group was

8 significantly lower than in the control group on days 1,3 and 10 and lower than in the

9 chorioamnionitis group on days 1-10.

10

11 Figure 2: Boxplots of Ang2/SC in TAF for small for gestational age infants (SGA) compared 12 to appropriate for gestational age infants (AGA). SGA infants had significantly higher 13 Ang2/SC on days 1-7 than AGA infants ( $\mathrm{p}<0.05$, respectively)

15 Figure 3: Boxplots of the ratio Ang1/Ang2 in TAF for the outcome moderate BPD or death. 16 The ratio was significantly decreased on days 3-10 for infants who developed BPD or died.

18 Figure 4: Mean concentrations with standard deviations of Ang1, Ang2 and sTIE-2 in

19 umbilical cord serum of infants who survived without BPD and infants who developed BPD

20 or died . sTIE-2 concentrations were significantly higher for infants who developed BPD or 21 died. 
Table 1: Clinical characteristics and short term outcome

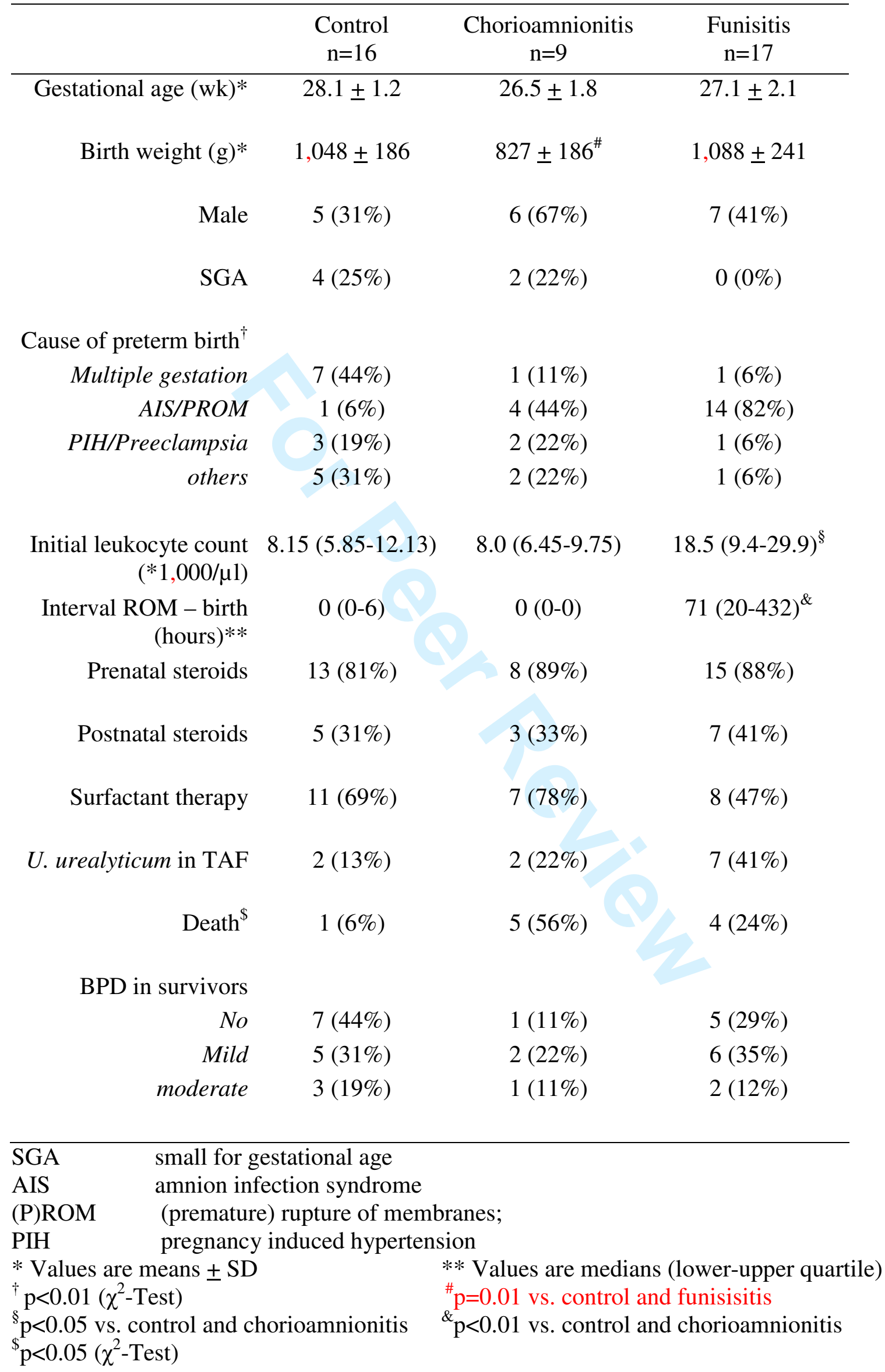


Reviewers' Comments to Author:

Reviewer: 2

Comments to the Author

Minor: page 5, include dates of enrollment of these infants.

The dates of enrollment were introduced in the revised version of the manuscript (page 5, line 5)

\section{page 6: rounds per minute (rpm) is usually referred to as revolutions per minute.}

The wording was changed according to the reviewers suggestion (page 6, line 7)

Reviewer: 1

Comments to the Author

Thomas and colleagues have presented a new version of the manuscript. I continue to have a few questions/issues that need to be addressed.

Major

This manuscript was difficult to read and understand the path to the conclusions that the authors reached. The authors seem to hang on to the need to try to link funisitis and Ang-Tie2 system. The authors have presented data that there is an association with increased Ang2/SC and BPD and with decreased Ang1/Ang2 ratio and BPD. It seems that funisitis does not correlate with either of these. SGA seems to be a potential confounder of these values and BPD. However, the authors go on to say none of these (Ang1/Ang2 or Ang2/SC) correlate with the development of BPD when the analysis was corrected for confounders of gestational age, SGA and gender. Also, the relationship between BPD and sTie2 became not significant when adjusted for gestational age. Yet the authors in their response to Reviewer 3 state "However, a limitation of this approach is also the power < $80 \%$ in most cases to detect a significant interaction between the confounders or an influence of the single confounder on the dependent parameter", so is it that the association with BPD is lost with the multivariate analysis or not? In other words, do the authors believe that they can preform a believable multivariate analysis with the small patient sample. If they do not believe that they have the power to perform such an analysis, then state that as a limitation of the study! This is a PILOT (preliminary) study correct? It is okay to have a PILOT study that suggests an association between an measurable factor and a clinical outcome. That is what drives the rationale to commit resources to the investigation/validation of the hypothesis generated by the PILOT study. In other words, state that these are PILOT data and the application of confounders for multivariate analysis led to a power of $<80 \%$ and thus could not be applied. This would lead to a 
rationale for a call for a larger study to look at the relationship between tracheal Ang2/SC and Angl/Ang2 ratio as a predictor of BPD. The authors may want to consider that they rewrite the manuscript as looking for an association between funisitis and Ang-Tie2 system and did not find that, but they found that Ang2/SC and Ang1/Ang2 ratio may be a predictor of BPD but a larger study with appropriate patient numbers to allow multivariate comparison for confounders must be done. They can use ref 9 (Aghai et al) to support their preliminary data about the role of Ang-Tie2 signaling is important to be considered in the risk for development of BPD.

We greatly appreciate the suggestions of the reviewer which helped us to improve the manuscript substantially.

With the approach of a multivariate analysis we followed what we understood as advice of reviewer 3 in the first review process. However, we agree with reviewer 1 that multivariate analysis with inclusion of potential confounders is of very limited value unless the power to detect associations and interactions is $>80 \%$. This is, as quoted from our former answer to reviewer 3, the case for most of the multivariate analyses we performed. We therefore decided to omit multivariate analysis from the second revision of our manuscript. We state that as major limitation in the discussion section (page 14, lines 18-25)

Following the reviewer's suggestion we state, that histological funisitis with the associated decrease of Ang2 in TAF, is most probably of minor relevance for outcome of the infants. In this regard we wrote our discussion more precisely. Indeed our preliminary data support the findings of Aghai and colleagues. We brought this issue into a sharper focus in the revised manuscript. We have changed the discussion section substantially for the second revision to be more stringent and precise.

\section{1- please consider showing the Ang2/SC data referred to on p9 line 18-20.}

We introduced the Ang2/SC values on day 10 for infants who developed moderate $B P D$ or died compared to those who survived without $B P D$ in the revised version of our manuscript (page 9, lines 18-20)

\section{2- Figure 4 does not seem needed and can be stated in the text.}

According to the reviewer's suggestion we omitted figure 4 from our revised manuscript. However, we found that presentation of individual data for Angl, Ang2 and sTIE2 for the three groups control, chorioamnionitis and funisitis in the text would be confusing for the reader. We therefore decided not to present them 
in the text. The new figure 4 (formerly figure 5) clearly depicts the range of values we measured for all 3 parameters.

\title{
3- Each figure should specify in the figure that the factor being represented is from Tracheal Aspirates or from umbilical cord blood.
}

\author{
The figures and legends were changed according to the reviewer's \\ suggestion
}

4- the authors allude to a relationship between Ang2 and VEGF on p13 and that increased Ang2 in the presence of decreased VEGF "causes endothelial cell death and vessel regression in the absence of sufficient angiogenic signals". Did they authors measure VEGF in their trach aspirate samples? If they did and there is a correlation between increased Ang2 and decreased VEGF, that would strongly support the assertion that there is an effect of impaired vascular signals on the risk of developing BPD.

We thank the reviewer for this very important remark. We did not measure VEGF in our samples. However, 2 groups have independently demonstrated VEGF protein levels in airway secretions to be lower in extremely premature infants who developed BPD compared to those who did not develop the disease (Lassus, AJRCCM 1999 and Been, Pediatr Res 2010). Another group demonstrated VEGF mRNA to be decreased in lungs of infants who died from BPD compared to controls who died from non-pulmonary causes (Bhatt, AJRCCM 2001). Therefore we still feel comfortable with our statement on page 13, lines 1-5 and we agree with the reviewer that low VEGF along with (relatively) increased Ang2 supports the concept of impaired vascular signaling in the pathogenesis of BPD which we state on page 12, Iines 22-24.

5- The authors try to make a link between intraamniotic Ang2 production and an increase in Ang2/SC at day 5 of life in the tracheal aspirates. This is quite a reach, I would expect that if there was a relationship between amniotic production and airway levels, that these should be elevated on the day of birth and day 1 . Perhaps the increased Ang2/SC is related to the treatments to keep a premature infant alive, e.g. Oxygen, ventilation, etc.

We are grateful for the reviewer's comment. We do not want to hypothesize that intraamniotic production of Ang2 might be a cause of elevated airway concentrations for this parameter on day 5 of postnatal life. The reviewer is absolutely right with his statement that rather postnatal factors like mechanical ventilation or oxygen therapy might contribute to increased release of Ang2 into the epithelial lining fluid of the infants. 
In normal gestations, Buhimschi demonstrated a direct correlation of GA with intraamniotic Angl and an inverse correlation with Ang2 along with an increase of the intraamniotic Angl/Ang2 ratio with increasing GA. This is thought to be mandatory for the integrity of the placental vasculature. Intraamniotic Angl and Ang2 is likely produced, at least to a certain extent, by the fetal lung (Buhimschi J Clin Endocrinol Metab 2010). Data on intramniotic Angl/Ang2 in $S G A$ infants or on pulmonary expression of the factors in utero do not exist to our knowledge. However it is an intriguing speculation that higher Ang2 as found on day 1-7 in our cohort (figure 2) and lower pulmonary Angl/Ang2 ratios might reflect a disturbed gestational regulation of of the angiogenic factors with effects for both impaired placental vascularization as in SGA infants and impaired pulmonary vascular development as in BPD. ( page 13, lines $5-16)$ 